\title{
Cyclic AMP-binding protein Epac1 acts as a metabolic sensor to promote cardiomyocyte lipotoxicity
}

\author{
Marion Laudette ${ }^{1,2}$, Yannis Sainte-Marie ${ }^{1}$, Grégoire Cousin ${ }^{1,3}$, Dorian Bergonnier ${ }^{1}$, Ismahane Belhabib ${ }^{1}$, Stéphanie Brun ${ }^{1,3}$, \\ Karina Formoso ${ }^{1}$, Loubna Laib ${ }^{1}$, Florence Tortosa ${ }^{1}$, Camille Bergoglio ${ }^{1}$, Bertrand Marcheix ${ }^{1,3}$, Jan Borén ${ }^{2}$, Olivier Lairez $\mathbb{D}^{1,3}$, \\ Jérémy Fauconnier ${ }^{4}$, Alexandre Lucas ${ }^{1}$, Jeanne Mialet-Perez (iD) ${ }^{1}$, Cédric Moro ${ }^{1}$ and Frank Lezoualc'h (iD) ${ }^{1}$
}

(c) The Author(s) 2021

Cyclic adenosine monophosphate (CAMP) is a master regulator of mitochondrial metabolism but its precise mechanism of action yet remains unclear. Here, we found that a dietary saturated fatty acid (FA), palmitate increased intracellular cAMP synthesis through the palmitoylation of soluble adenylyl cyclase in cardiomyocytes. CAMP further induced exchange protein directly activated by cyclic AMP 1 (Epac1) activation, which was upregulated in the myocardium of obese patients. Epac1 enhanced the activity of a key enzyme regulating mitochondrial FA uptake, carnitine palmitoyltransferase 1. Consistently, pharmacological or genetic Epac1 inhibition prevented lipid overload, increased FA oxidation (FAO), and protected against mitochondrial dysfunction in cardiomyocytes. In addition, analysis of Epac1 phosphoproteome led us to identify two key mitochondrial enzymes of the the $\beta$-oxidation cycle as targets of Epac1, the long-chain FA acyl-CoA dehydrogenase (ACADL) and the 3-ketoacyl-CoA thiolase (3-KAT). Epac1 formed molecular complexes with the $\mathrm{Ca}^{2+} /$ calmodulin-dependent protein kinase II (CaMKII), which phosphorylated ACADL and 3-KAT at specific amino acid residues to decrease lipid oxidation. The Epac1-CaMKII axis also interacted with the a subunit of ATP synthase, thereby further impairing mitochondrial energetics. Altogether, these findings indicate that Epac1 disrupts the balance between mitochondrial FA uptake and oxidation leading to lipid accumulation and mitochondrial dysfunction, and ultimately cardiomyocyte death.

Cell Death and Disease (2021)12:824; https://doi.org/10.1038/s41419-021-04113-9

\section{INTRODUCTION}

The heart has a high energy demand and most of the ATP produced comes from mitochondrial oxidative phosphorylation, from which fatty acid oxidation (FAO) contributes to $70 \%$ of the energy supply [1]. In metabolic diseases such as obesity and type 2 diabetes mellitus, accumulation of intramyocardial lipids contributes to cardiac defects termed lipotoxic cardiomyopathy [2]. In this cardiac insult, the metabolic flexibility of the myocardium is compromised as illustrated by an increase in the use of fatty acids (FA) as an energy substrate, and a decrease in carbohydrates utilization [3]. In addition, an imbalance between FA uptake and oxidation results in intracellular accumulation of lipid intermediates thereby causing reactive oxygen species (ROS) generation, mitochondrial dysfunction, and cardiomyocyte death [2]. This metabolic alteration contributes to cardiac alteration [4]. Therefore, impaired myocardial metabolism and mitochondrial dysfunction play a critical role in the pathogenesis of lipotoxic cardiomyopathy. However, how myocardial FA metabolism is disrupted during lipotoxic insults yet remains elusive.

Cyclic adenosine monophosphate (CAMP) is a versatile signaling molecule that regulates a large variety of cellular processes, including cardiac contraction, cellular death, and metabolism [5].
Synthesis of CAMP from adenosine triphosphate (ATP) is generated by membrane-bound adenylyl cyclases (AC) upon activation of G-coupled-protein receptors (GPCR) [6]. The type 10 soluble adenylyl cyclase (sAC) constitutes the second source of CAMP and is activated by $\mathrm{HCO}_{3}{ }^{-}$and $\mathrm{Ca}^{2+}$ to control various cellular processes $[7,8]$. In mitochondria, SAC promotes posttranslational regulation of mitochondrial oxidative phosphorylation activity via CAMP-dependent protein kinase A (PKA) phosphorylation to stimulate ATP synthesis [9-11]. Interestingly, previous findings reported that the sympathetic nervous system, which is a key regulator of cardiac function, is overstimulated in the diabetic heart and contributes to impaired CAMP-PKA signaling $[12,13]$. However, little is known on the role and mechanisms of CAMP action in the control of myocardial metabolism and lipotoxicity induced by lipid overload.

Although PKA has long been considered as the most active kinase in the mitochondrial matrix and the main effector of intramitochondrial CAMP [14], the CAMP downstream target, exchange protein directly activated by CAMP 1 (Epac1) has recently gained a lot of interest for its mitochondrial localization and role [15-17]. Epac1 is a guanine-nucleotide-exchange factor (GEF) that mediates the CAMP-dependent but PKA-independent activation of

\footnotetext{
${ }^{1}$ Institut des Maladies Métaboliques et Cardiovasculaires, Inserm, Université Paul Sabatier, UMR 1297-I2MC, Toulouse, France. ${ }^{2}$ Department of Molecular and Clinical Medicine,

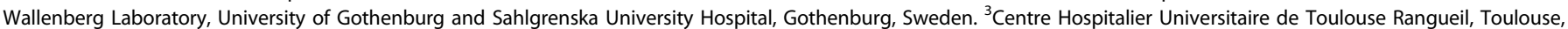
France. ${ }^{4}$ PHYMEDEXP, Université de Montpellier, CNRS, INSERM, CHRU Montpellier, Montpellier, France. ${ }^{\bowtie}$ email: Frank.Lezoualch@inserm.fr Edited by Professor Alessandro Finazzi-AgrÃ
} 
small G-proteins of the Ras superfamily, Rap1 and Rap2 [18, 19]. Compelling evidence is now accumulating about the formation of Epac1 molecular complexes in distinct cellular sites that influence Epac1 signalling and cellular function $[17,20]$. With respect to the mitochondrial compartment, Epac1 regulated the dynamic phosphorylation of dynamin-1-like protein (DRP1), which is crucial for mitochondrial fission/fusion balance [21]. Another study demonstrated that, during cardiac ischemia, mitochondrial Epac1 increased $\mathrm{Ca}^{2+}$ uptake and ROS production thereby promoting mitochondrial death signalling such as mitochondrial permeability transition pore (MPTP) opening and cytochrome $c$ release, and ultimately cardiomyocyte death [16]. While it is clear that Epac1 is expressed in mitochondria, further studies are needed to determine mitochondrial Epac1 molecular complexes and biological function.

Here, we report that a diet-dependent signal, palmitate regulates the intracellular synthesis of CAMP to activate Epac1, which in turn promotes lipid accumulation and mitochondrial dysfunction. We unravel an unsuspected molecular mechanism by which CAMP-Epac1 signaling induces metabolic inflexibility and contributes to lipotoxicity in cardiomyocytes.

\section{RESULTS \\ Palmitic acid stimulates mitochondrial sAC to activate cAMP- Epac1 signaling}

Palmitate is a major saturated FA in the plasma, and chronic exposure of primary cardiomyocytes to bovine serum albumin (BSA)-bound palmitic acid produces a model of FA-induced lipotoxicity in vitro [22]. Using the LDH and MTT assays, we found that one day treatment with palmitate promoted cardiomyocyte death Epac1 (Fig. 1A, Supplementary Fig. 1). This effect was blocked by a selective Epac1 inhibitor CE3F4 [23], suggesting that palmitate activated Epac1 (Fig. 1A, Supplementary Fig. 1). Accordingly, the Epac1 specific agonist, 8-pCPT-2'-O-Me-cAMPAM (8-CPT-AM) [24] failed to potentiate the effect of palmitate on cardiomyocyte death (Fig. 1A, Supplementary Fig. 1). Of note, palmitate increased the amount of intracellular cAMP, the cognate activator of Epac1 and thus the active form of Rap1, a direct effector of Epac1 (Fig. 1B and Supplementary Fig. 2A). As expected, a nontoxic FA (Supplementary Fig. 2B), oleate failed to induce Rap1 activation (Supplementary Fig. 2C). Furthermore, a selective SAC inhibitor, KH7, blocked CAMP accumulation and significantly reduced palmitate-induced Epac1 activation in a manner similar to CE3F4 (Fig. 1B). Altogether, these data demonstrate that palmitate stimulates $\mathrm{SAC}$ and subsequent CAMP production promotes Epac1 activation, which triggers cardiomyocyte death.

We used a nonradioactive acyl-biotin exchange (ABE) method to determine how palmitate regulates SAC activity [25]. Treatment with a reducing agent, hydroxylamine $\left({ }^{\prime \prime}+{ }^{\prime \prime}\right)$ reveals proteins initially palmitoylated and captured by streptavidin before cleavage. The absence of signal in "-" lane is a negative control corresponding to all initially free cysteines that have been blocked. It confirms that the signal obtained in " + " lane depends on the activity of hydroxylamine and reflects the state of palmitoylation of the protein. We found that both Epac1 and mitochondrial SAC were palmitoylated under basal condition (Fig. 1C). However, palmitate enhanced sAC palmitoylation but not that of Epac1 (Fig. 1C). Bioinformatic analysis of mitochondrial SAC sequence revealed a potential palmitoylation site on Cys342 of $s A C$, which is located in the $\mathrm{C} 2$ catalytic domain of $\mathrm{SAC}$, close to the ATP and $\mathrm{HCO}_{3}{ }^{-}$ binding sites [26] and is phylogenetically conserved across SAC orthologs (Fig. 1D and Supplementary Fig. 2D). Remarkably, a mutant SAC with this Cys residue substituted to Ala residue $\left(s A C^{C 342 A}\right.$ ) mutant failed to be palmitoylated compared to $S A C^{W T}$ in palmitate-treated cardiomyocytes (Fig. 1E). This effect of $s A C^{C 342 A}$ translated on CAMP production and Rap1-GTP (Fig. 1F, G).
Accordingly, palmitate-induced cardiomyocyte death was compromised in $S A C^{C 342 A}$ transfected cells (Fig. 1H). Altogether, these data provide strong evidence that mitochondrial SAC is posttranslationally activated by palmitoylation to promote Epac1induced cardiomyocyte death.

\section{Epac1 impairs mitochondrial function during a chronic lipotoxic stress}

While chronic exposure of palmitate reduced maximal mitochondrial respiration in wild-type (WT) cardiomyocytes, Epac1 ${ }^{-1-}$ cardiomyocytes were resistant to palmitate-induced oxygen consumption rate (OCR) decrease (Fig. 2A, B). In addition, palmitate provoked an increase in mitochondrial ROS (mROS) content assayed with the superoxide sensitive probe MitoSOX Red; superoxide production remained low and similar to control condition in cells devoid of Epac1, or in WT cardiomyocytes treated with CE3F4 (Fig. 2C). Consistently, Epac1 genetic or pharmacological inhibition prevented palmitate-induced loss of mitochondrial membrane potential $(\Delta \Psi \mathrm{m})$ in isolated cardiomyocytes (Fig. 2D). Direct activation of Epac1 with 8CPT-AM had similar effect of palmitate on mitochondrial dysfunction (Supplementary Fig. 3A, B) and cardiomyocyte apoptosis (Supplementary Fig. 3C). Of note, Epac1 pharmacological inhibitors and palmitate failed to dysregulate the expression level of oxidative phosphorylation enzyme complexes indicating that these compounds did not induce lesions of the oxidative phosphorylation system in our experimental conditions (Supplementary Fig. 4). Finally, cardiomyocytes transfected with a mutant form of Epac1 deleted for its putative mitochondrial-targeting sequence (Epac1 $\left.{ }^{\Delta 2-37},[16]\right)$ exhibited less mROS and caspase 3 activation compared to those transfected with the Epac1 ${ }^{\mathrm{WT}}$ and treated with palmitate (Fig. 2E, F). As expected, palmitate-induced cell death was reduced in Epac1 ${ }^{\Delta 2-37}$ expressing cardiomyocytes (Fig. 2G). Together, these data strongly suggest that mitochondrial expression of Epac1 promotes mitochondrial dysfunction and cardiomyocyte death during persistent lipid overload.

\section{Epac1 promotes mitochondrial FA uptake}

Obesity increases circulating levels of free FAs that accumulate in the myocardium to promote cardiomyocyte lipotoxicity [2]. Epac1 expression level was upregulated in the myocardium of obese patients compared with that of controls (Fig. 3A and Supplementary Fig. 5A). Similarly, cardiac samples from mice subjected to a high-fat diet (HFD, 60\% lipid) for a long term period of 9 months had increased Epac1 expression. Interestingly, Epac1 inhibition with CE3F4 reduced lipid overload in primary cardiomyocytes incubated with palmitate (Fig. $3 \mathrm{C}$ and Supplementary Fig. 5B). Because CaMKII was reported to act as a downstream effector of Epac1 in various biological processes [17, 20], we investigated the effect of a CaMKII pharmacological inhibitor, KN-93 on lipid overload. As expected, KN-93 mimicked the effect of CE3F4 on lipid accumulation (Fig. 3C and Supplementary Fig. 5B). The Epac1 agonist, 8-CPT-AM failed to potentiate the effect of palmitate (Fig. 3C) because this FA behaved as a stimulator of Epac1 (Fig. 1B). Interestingly, Epac1 ${ }^{\text {WT }}$ transfected cells displayed increased lipid accumulation than those overexpressing the mutant Epac1 ${ }^{\triangle 2-37}$ that excluded Epac1 from the mitochondria [16] suggesting that cytosolic Epac1 regulates lipid accumulation and/or mitochondrial Epac1 controls FA metabolism (Fig. 3D).

Long-chain FAs are transported across the mitochondrial membrane by the carnitine palmitoyltransferase 1 (CPT-1) [27]. Thus, CPT-1 plays a critical role in mitochondrial FA uptake and constitutes a rate-limiting enzyme in FA oxidation. We therefore addressed whether Epac1 would regulate FA transport inside mitochondria via CPT-1 activity. To test this hypothesis, we measured mitochondrial OCR after a short-term treatment with palmitate and in the presence or absence of a CPT-1 inhibitor, etomoxir (Fig. 3E). As previously described [28], acute stimulation with palmitate provoked an increase in OCR in primary 

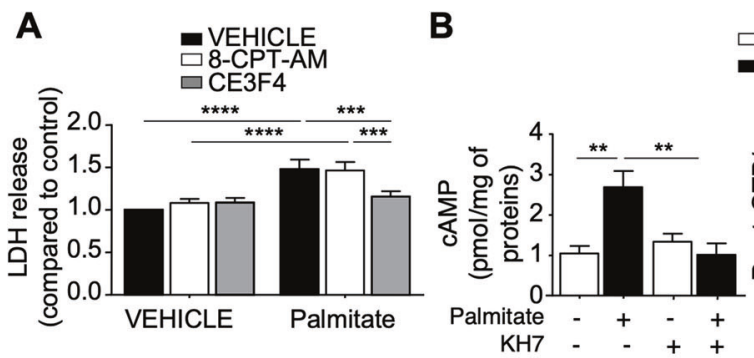

$\square$ VEHICLE

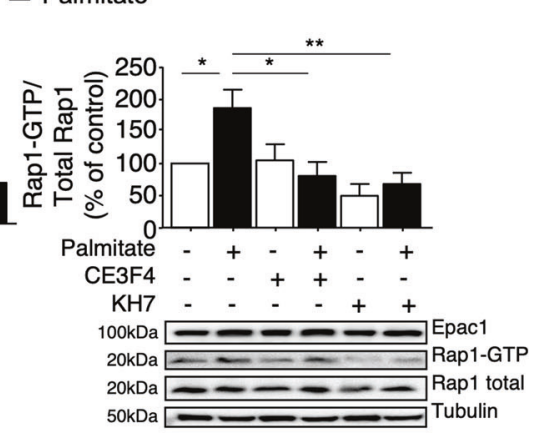

C
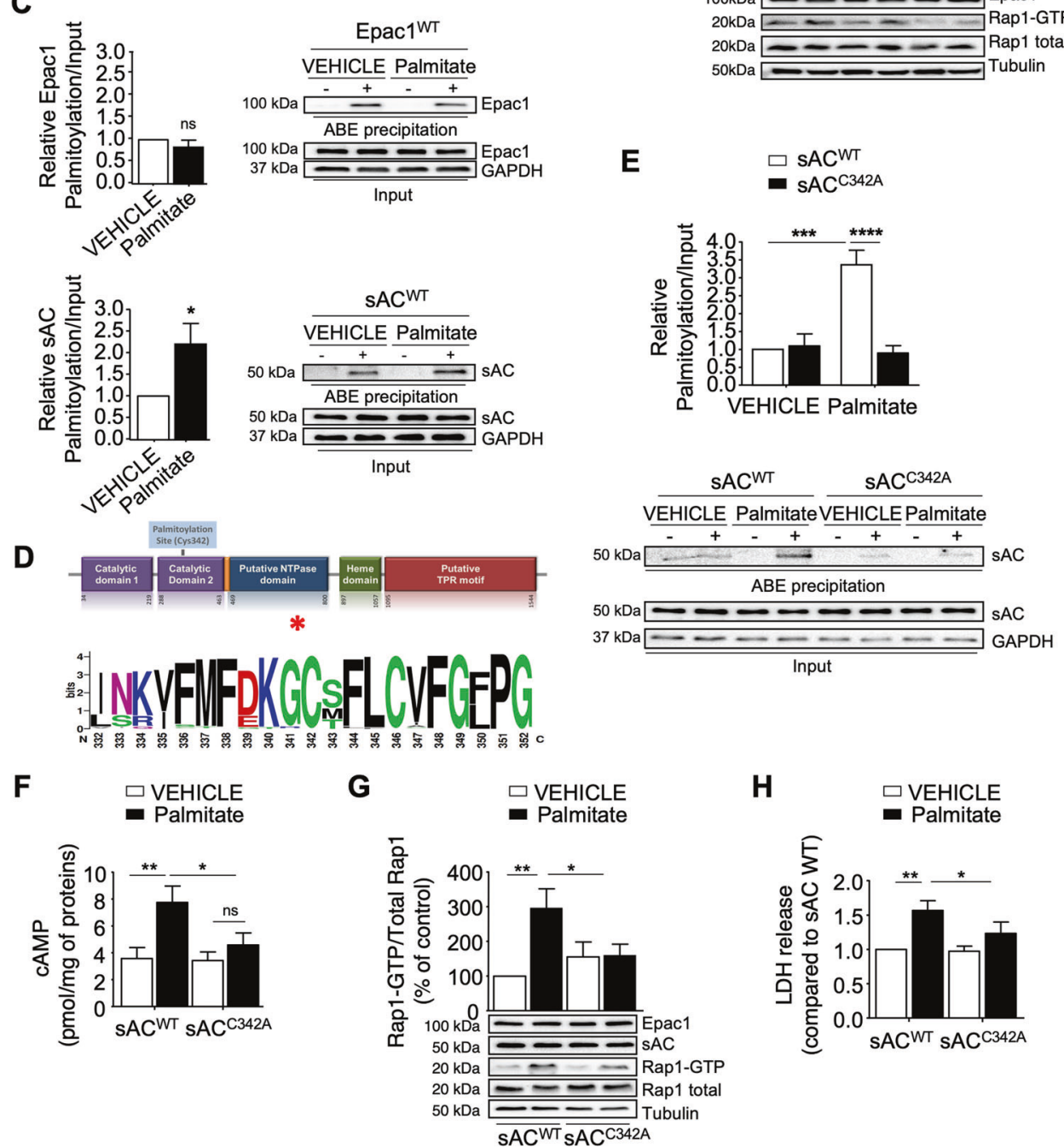

Fig. 1 Palmitate treatment increases CAMP production and Epac1 activity through SAC palmitoylation. A Measurement of LDH release in cardiomyocytes pretreated with 8-CPT-AM $(10 \mu \mathrm{M}, 30 \mathrm{~min})$ or CE3F4 $(20 \mu \mathrm{M}, 30 \mathrm{~min})$ and incubated with either palmitate $(500 \mu \mathrm{M}, 24 \mathrm{~h})$ or its VEHICLE (BSA-control) $(n=6)$. B Intracellular CAMP $(n=6)$ and Rap1-GTP $(n=6)$ levels in cardiomyocytes transfected with Epac1 and pretreated or not with CE3F4 $(20 \mu \mathrm{M}, 30 \mathrm{~min})$ or $\mathrm{KH} 7(20 \mu \mathrm{M}, 30 \mathrm{~min})$ before incubation with palmitate $(500 \mu \mathrm{M}, 1 \mathrm{~h})$. C, E Quantification of Epac1 $(n=5)$, mitochondrial sAC $\left(s A C^{W T}\right)(n=6)$ or a mutant $s A C^{C 342 A}$ palmitoylation in cells transfected with the indicated plasmids and treated with palmitate $(500 \mu \mathrm{M}, 1 \mathrm{~h})(n=4)$. D Scheme of the functional domains in mammalian sAC and graphical representation with Weblogo software of a multiple sequence alignment of 97 sequences of sAC from different species performed with Consurf software. The mammalian SAC is comprised of two catalytic domains C1 and C2 (violet) localized in the N-terminal domain, followed by an autoinhibitory motif (orange). The C-terminal region contains a putative NTPase domain (blue), an Heme domain (green) and putative TPR modules (red). Within the C2 there is a putative palmitoylation site (C342). F-H Intracellular CAMP $(\mathbf{F}, n=6)$ or Rap1-GTP $(\mathbf{G}, n=6)$ levels, LDH release $(\mathbf{H}, n=$ 7) in cells transfected with the indicated plasmids. The results are expressed as mean \pm SEM and were analysed with a two-way ANOVA/ Bonferroni post test. ${ }^{*} p<0.05,{ }^{* *} p<0.01,{ }^{* * *} p<0.001,{ }^{* * * *} p<0.0001$ vs indicated value. Representative immunoblots are shown.

cardiomyocytes, an effect that was inhibited by etomoxir (Fig. 3E, F). Similarly, pretreatment of cells with CE3F4 prevented palmitateinduced augmentation in mitochondrial respiration (Fig. 3E, F). In agreement with these results, 8-CPT-AM significantly increased
CPT-1 activity, which was blocked by either etomoxir or CE3F4 (Fig. 3G). Altogether, these results demonstrate that Epac1 increases CPT-1 activity to promote FA uptake inside mitochondria (Fig. 3H). 
A

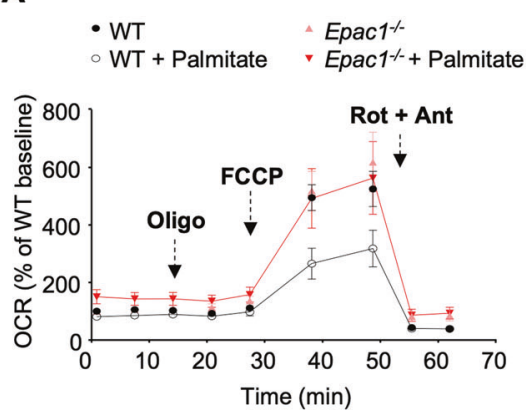

C

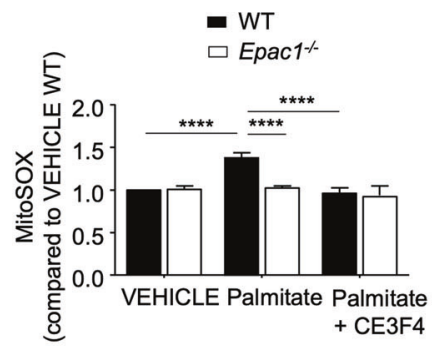

B

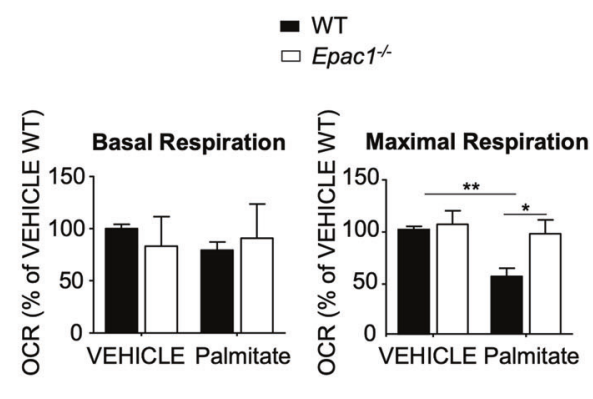

D

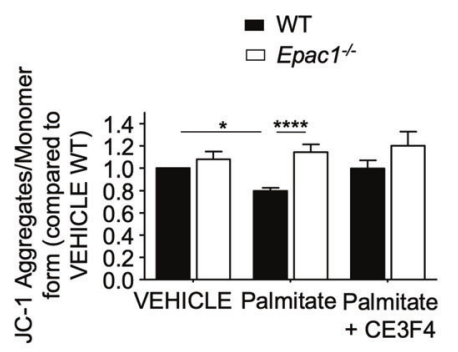

G

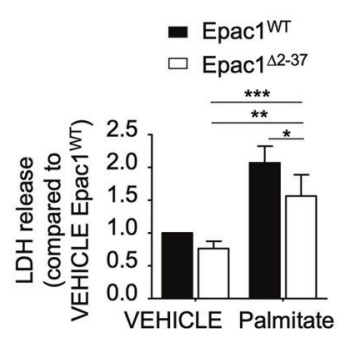

Fig. 2 Epac1 promotes mitochondrial aberrations leading to cardiomyocyte death. A Measurements of oxygen consumption rate (OCR) in adult cardiomyocytes isolated from WT or Epac1 ${ }^{-1-}$ mice, incubated or not (VEHICLE) with palmitate $(150 \mu \mathrm{M}, 2 \mathrm{~h})$ after oligomycin, FCCP, antimycin A or rotenone $(1 \mu \mathrm{M})$ addition $(n=4-5)$. B Graphical representation of basal or maximal respiration of WT and Epac1 ${ }^{-\prime-}$ cardiomyocytes subjected to palmitate-induced metabolic stress $(n=4-5)$. C, D Quantification of mitochondrial ROS using MitoSOX (C) and mitochondrial membrane potential (D) using JC-1 probe in WT or Epac1 ${ }^{-1-}$ cardiomyocytes pretreated with CE3F4 (20 $\mu M_{1} 30$ min) or (VEHICLE) and stimulated with palmitate $(150 \mu \mathrm{M}, 2 \mathrm{~h})(n=6)$. E-G Primary cardiomyocytes expressing wild-type Epac1 (Epac1 $\left.{ }^{\text {WT }}\right)$ or mutant deleted for its putative mitochondrial-targeting sequence (Epac1 $\left.{ }^{\Delta 2-37}\right)$ were stimulated with 8 -CPT-AM (10 $\mu \mathrm{M}, 30$ min) and incubated with palmitate $(500 \mu \mathrm{M})$. Mitochondrial ROS quantification $(\mathbf{E}, n=7)$, caspase 3 activity $(\mathbf{F}, n=5)$, or LDH release $(\mathbf{G}, n=11)$. Results are expressed as mean \pm SEM and were analyzed with a two-way ANOVA/Bonferroni post test. ${ }^{*} p<0.05,{ }^{* *} p<0.01,{ }^{* * *} p<0.001,{ }^{* * * *} p<0.0001$ vs indicated value.

\section{Epac1 inhibits metabolic flexibility of cardiomyocytes}

Since mitochondrial FAO capacity is coupled with FA mitochondria uptake [29], we next assessed whether Epac1 would influence palmitate oxidation by measuring the degree of $\mathrm{CO}_{2}$ produced from the degradation of radiolabelled $\left[{ }^{14} \mathrm{C}\right]$-palmitate in cardiomyocytes. Cells treated with either CE3F4 or KN-93 had increased amount of radiolabelled $\mathrm{CO}_{2}$ indicating an inhibitory effect of Epac1/CaMKII axis on FAO (Fig. 4A). As expected, direct Epac1 activation with 8-CPT-AM failed to influence $F A O$ because of the stimulating effect of palmitate on Epac1 activation (Fig. 1B). FAO levels were similar in vehicle and CE3F4 treated cardiomyocytes suggesting that Epac1 was not involved in FA metabolism in basal condition (Fig. 4B, C; Supplementary Fig. 6A). On the contrary, pharmacological inhibition of Epac1 with CE3F4 significantly increased FAO levels in palmitatetreated cardiomyocytes (Fig. 4B, D). We used FCCP to measure the ability of cells to respond to increased energy demand (leading to a rapid oxidation of palmitate in this context) and found that CE3F4 enhanced maximal respiration as well as respiratory capacity in FCCPinduced stress conditions compared to untreated cells. These data demonstrate Epac1 inhibition allows cardiomyocytes to better cope with increased energy supply linked to an excess of FA (Fig. 4B, E; Supplementary Fig. 6B).

Next, we sought to determine whether Epac1 also played a role in glucose oxidation. We measured the ExtraCellular Acidification Rate (ECAR) of cardiomyocytes using Seahorse XF24 flow analyzer in the presence of glucose, oligomycin (ATP synthase inhibitor to enhance glycolysis), or 2-deoxyglucose (glycolysis inhibitor). After addition of a saturating concentration of glucose, 8-CPT-AM tended to decrease glycolysis while CE3F4 increased ECAR indicating that Epac1 inhibition enhanced the glycolytic capacity of cardiomyocytes (Supplementary Fig. 6C, D). Of particular importance, metabolic potential measurements using Seahorse Cell Energy Phenotype Test (to determine basal and maximal mitochondrial respiration and glycolytic activity in living cells) showed that palmitate significantly increased the oxidative metabolic potential (OCR) of cardiomyocytes without affecting their glycolytic metabolic potential (ECAR) (Fig. 4E, F). Strikingly, cells treated with CE3F4 exhibited a higher oxidative and 
A

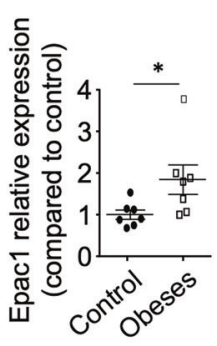

C

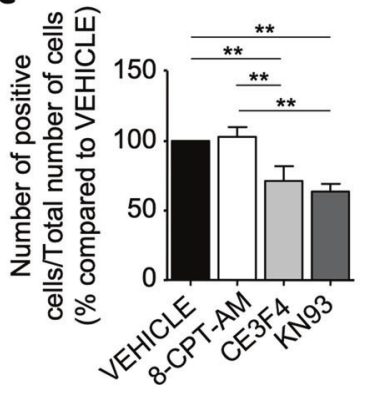

E

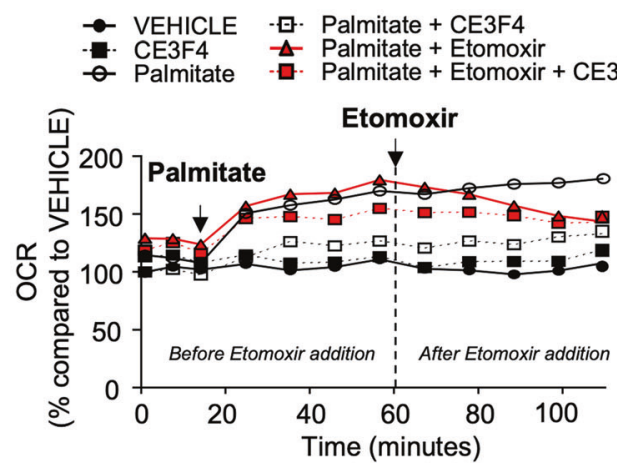

D
B

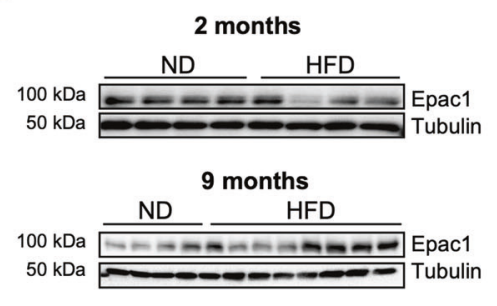

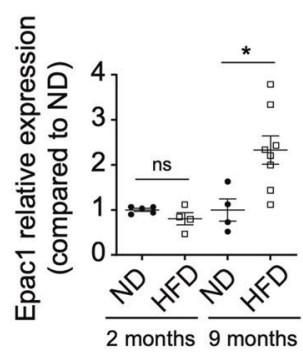

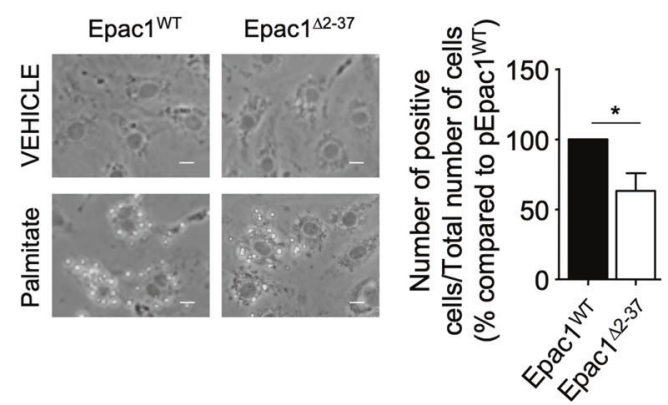

F

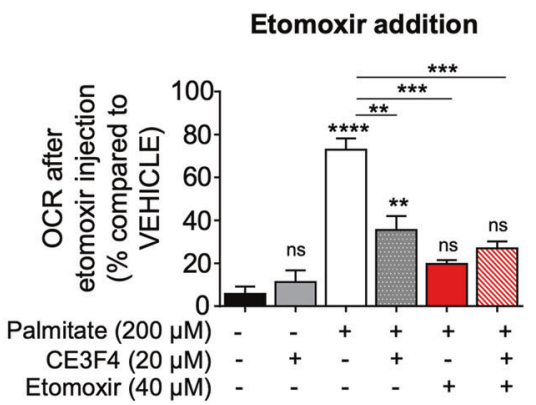

H
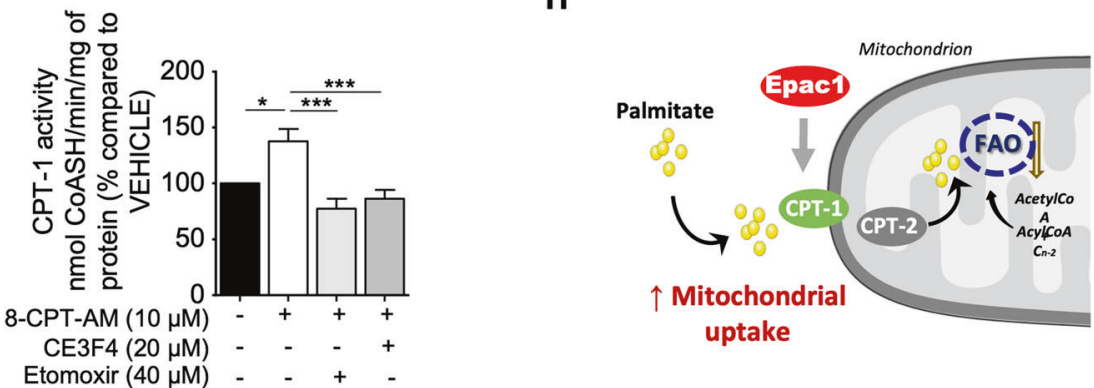

Fig. 3 Epac1 promotes lipid accumulation and regulates mitochondrial FA uptake. A, B Representative immunoblot and quantification of Epac1 protein in human right atrial appendage tissues from non-obese control or obese patients $(n=7)$, and in cardiac samples from C57Bl6 mice subjected to normal diet (ND) or high-fat diet (HFD) for 2 or 9 months $(n=5-8)$. GAPDH, Tubulin, loading control. C, D Quantification of lipid accumulation (C) in primary cardiomyocytes pretreated or not (VEHICLE) with 8-CPT-AM (10 $\mu$ M, $30 \mathrm{~min}), \mathrm{CE} 3 \mathrm{~F} 4(20 \mu \mathrm{M}, 30 \mathrm{~min})$, or KN-93 $(5 \mu \mathrm{M}, 30 \mathrm{~min})$ and incubated with palmitate $(500 \mu \mathrm{M}, 24 \mathrm{~h})(n=4-6$ different experiments) Scale, $10 \mu \mathrm{m}$. In D cells were transfected with the indicated plasmids for $24 \mathrm{~h}$ and then incubated with palmitate $(500 \mu \mathrm{M}, 24 \mathrm{~h})(n=5$ different experiments). Representatives images are shown on the left panel. E, F Measurement of OCR in cardiomyocytes pretreated with CE3F4 (20 $\mu \mathrm{M}, 30 \mathrm{~min})$ or not (VEHICLE) after acute treatment of palmitate $(500 \mu \mathrm{M}, 15 \mathrm{~min})$ and CPT-1 inhibitor, etomoxir $(40 \mu \mathrm{M}, 60 \mathrm{~min})(n=2-4)$. In $\mathbf{F}$ the graphical representation is shown $(n=2-4)$. G CPT-1 activity in cardiomyocytes pretreated or not with 8 -CPT-AM $(10 \mu \mathrm{M}, 30 \mathrm{~min})$ and then treated with CE3F4 (20 $\mu \mathrm{M}, 30 \mathrm{~min})$ or etomoxir $(40 \mu \mathrm{M}, 30 \mathrm{~min})(n=6-8)$. H Schematic representation of the regulation of CPT-1 activity by Epac1. The results are expressed as mean \pm SEM and were analyzed with a two-way ANOVA/Bonferroni post test. In A, a Mann-Whitney test was performed. ${ }^{*} p<0.05,{ }^{* *} p<0.01,{ }^{* * *} p<0.001$, ${ }_{* * * *} p<0.0001$ vs indicated value. ns not significant. 
A

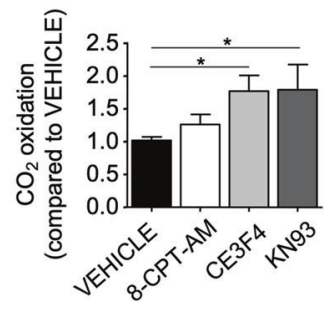

C
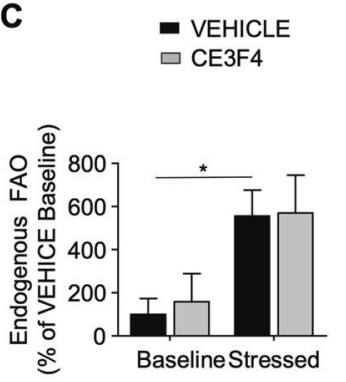

B

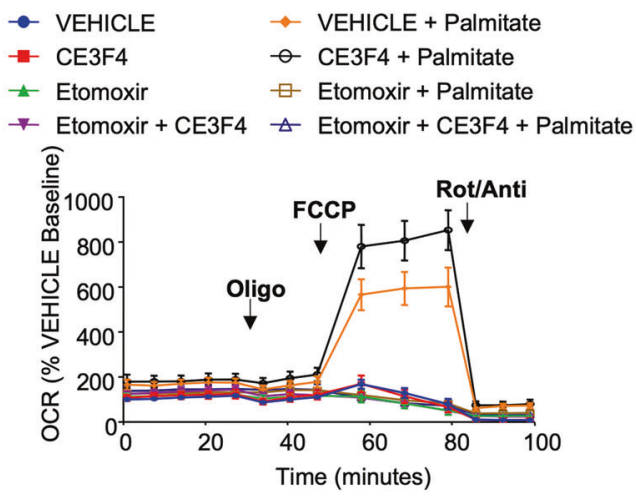

D
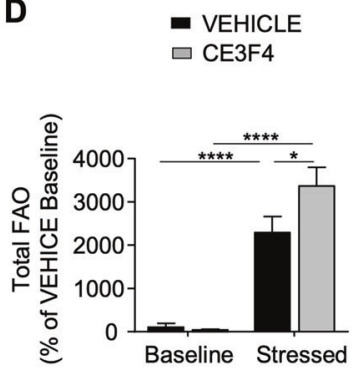

$\mathbf{E}$

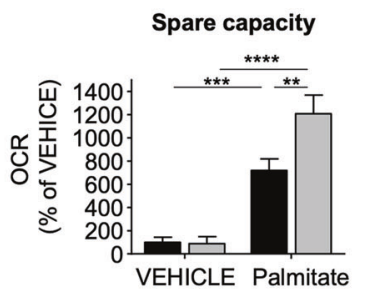

$\mathbf{F}$
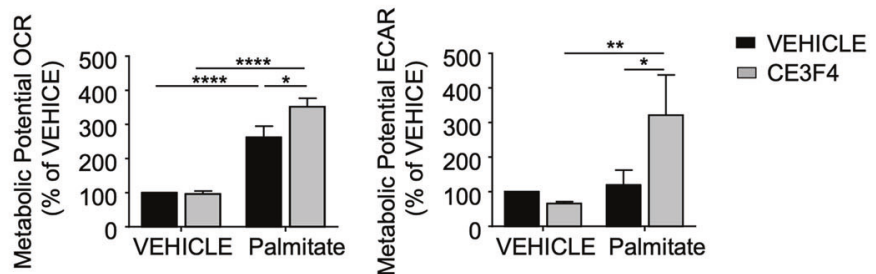

Fig. 4 Epac1 regulates FA and carbohydrates oxidation. A Determination of $\mathrm{FAO}$ in cardiomyocytes by measuring radiolabeled $\mathrm{CO}_{2}$ produced by palmitate ${ }^{14} \mathrm{C}$. Cells were pretreated or not (VEHICLE) with 8 -CPT-AM $(10 \mu \mathrm{M}, 30 \mathrm{~min}), \mathrm{CE} 3 \mathrm{~F} 4(20 \mu \mathrm{M}, 30 \mathrm{~min})$, or KN-93 $(5 \mu \mathrm{M}$, $30 \mathrm{~min}$ ) before addition of palmitate ${ }^{14} \mathrm{C}$ for $3 \mathrm{~h}(n=9)$. B Measurement of OCR in cardiomyocytes pretreated with CE3F4 (20 $\left.\mu \mathrm{M}, 30 \mathrm{~min}\right)$ or not (VEHICLE) after oligomycin $(2 \mu \mathrm{M}), \mathrm{FCCP}(2 \mu \mathrm{M})$, antimycin $\mathrm{A}(4 \mu \mathrm{M})$, and rotenone $(2 \mu \mathrm{M})$ addition $(n=7)$. Cells were pretreated with etomoxir $(40 \mu \mathrm{M}, 15 \mathrm{~min})$ before palmitate addition $(500 \mu \mathrm{M})$ at time 0 . C, D Quantification of endogenous (C) or D total (endogenous + exogenous) oxidation of FA in baseline and in FCCP-induced stress conditions. E Graphical representation of respiratory capacity of cardiomyocytes pretreated with CE3F4 $(20 \mu \mathrm{M}, 30 \mathrm{~min})$ or not (VEHICLE) and treated with palmitate $(500 \mu \mathrm{M})$. F Metabolic Potential calculated by the Seahorse XF Cell Energy Phenotype Test Report Generator (Percentage of stressed (FCCP addition) OCR or ECAR over baseline OCR or ECAR. This parameter is the measure of cells' ability to meet an energy demand via respiration and glycolysis. The results are expressed as mean \pm SEM and were analyzed with a two-way ANOVA/Bonferroni post test. ${ }^{*} p<0.05,{ }^{* *} p<0.01,{ }^{* * *} p<0.001,{ }^{* * * *} p<0.0001$ vs indicated value.

glycolytic metabolic potential indicating that Epac1 inhibition prevented FAO-induced glucose oxidation inhibition in FA overload condition (Fig. 4E, F). Altogether, these results show that Epac1 inhibition allows a better metabolic flexibility of cardiomyocytes in the context of excess lipid flux.

\section{Epac1 targets $\beta$-oxidation promoting enzymes through a CaMKII-dependent pathway}

To further elucidate the molecular mechanisms whereby Epac1 influenced energy metabolism, we next searched for molecular targets of Epac1. We analyzed data from Epac1 phosphoproteome that we performed using two-dimensional electrophoresis coupled to tandem mass spectrometry [16]. Among the phosphopeptides identified, spot 127 and spot 155 corresponded to two mitochondrial enzymes, the long-chain fatty acid acyl-CoA dehydrogenase (ACADL) and the 3-ketoacyl-CoA thiolase (3-KAT) (Fig. 5A, Supplementary Fig. 7A). ACADL and 3-KAT catalyze the first and last key steps in mitochondrial FAO, respectively [1]. In line with the Epac1 phosphoproteome analysis, co-immunoprecipitation experiments showed that Epac1 activation increased ACADL and 3-KAT serine-specific phosphorylation (Fig. 5B and Supplementary Fig. 7B). Furthermore, mitochondrial Epac1, CaMKII, and ACADL or 3-KAT were involved in the same macromolecular complex (Fig. 5C and Supplementary Fig. 5B). Pharmacological inhibition of Epac1 (CE3F4) or CaMKII (KN-93) prevented Epac1-mediated ACADL or 3-KAT phosphorylation (Fig. 5D and Supplementary Fig. 7C).

Interestingly, a bioinformatic analysis of ACADL sequence identified a potential phosphorylation site on Ser193 for CaMKII isoform. Knockdown of CaMKII $\delta$ isoform with specific siRNA (SiCaMKIII) blocked 8-CPT-AM-induced ACADL phosphorylation, demonstrating that CaMKII $\delta$ specifically targets ACADL in response to Epac1 activation (Fig. 5E). Of note, palmitate promoted ACADL phosphorylation was prevented by CE3F4 suggesting that palmitate via the SAC-CAMP-Epac1 axis regulates the phosphorylation state of ACADL (Fig. 5F). Transfection of a mutant ACADL with Ser193 substituted to Ala residue (ACADL ${ }^{\text {S193A }}$ ) blocked 8-CPT-AM or palmitate mediated ACADL serine phosphorylation compared to $\mathrm{ACADL}^{\mathrm{WT}}$ (Fig. 5H, I). Importantly, cells expressing ACADL ${ }^{\mathrm{S} 193 \mathrm{~A}}$ were 
A

\begin{tabular}{|c|c|}
\hline Spot ID & 127 \\
\hline Ratio (Vol) & 4,48 \\
\hline Regulation & Up \\
\hline ProteinID & $\begin{array}{c}\text { Long-chain specific } \\
\text { acyl-CoA } \\
\text { dehydrogenase, } \\
\text { mitochondrial }\end{array}$ \\
\hline Score & 244 \\
\hline
\end{tabular}

D

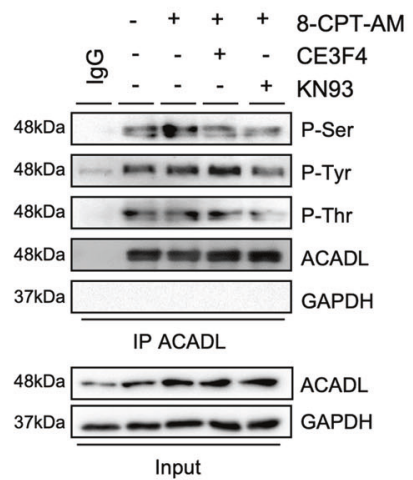

G
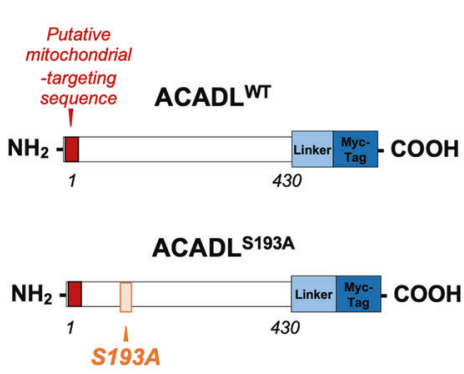

I

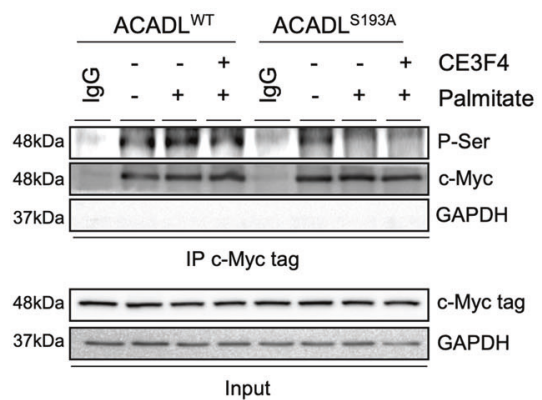

C

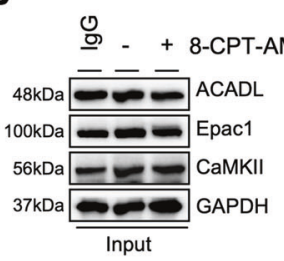

E

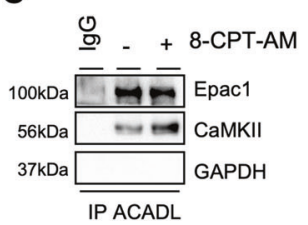

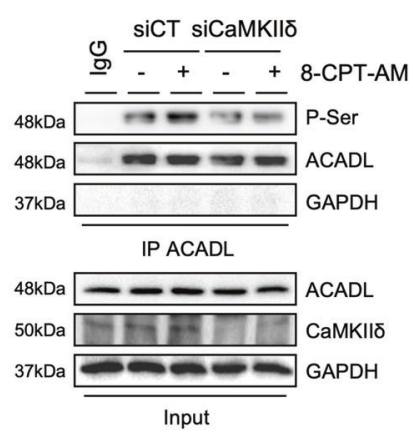

F

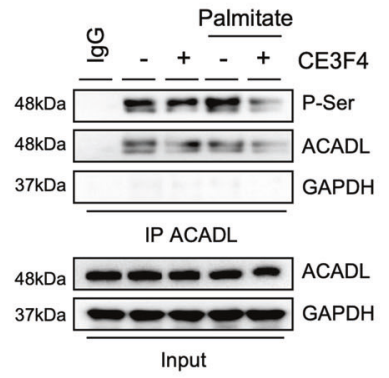

H

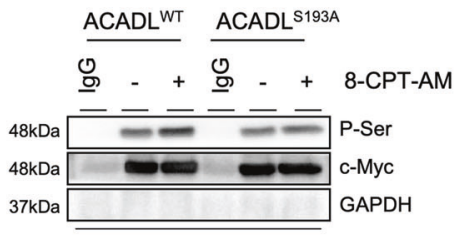

IP c-Myc tag

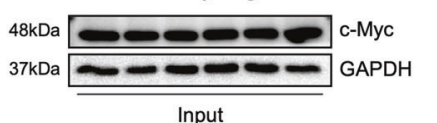

J

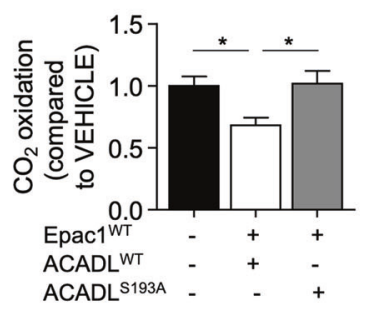

Fig. 5 Epac1-CaMKII targets Acyl-CoA dehydrogenase (ACADL) to decrease FA breakdown. A Phosphoproteome results. The ratio of volume value is indicated in the table. The change in the intensity of the protein spot (127) is indicated as increased (up) in the stimulated 8-CPT-AM (10 $\mu \mathrm{M}, 10 \mathrm{~min})$ vs. control. ID identity. B Representative immunoblots showing ACADL phosphorylation at Ser, Thr, and Tyr residues in cardiomyocytes stimulated or not with 8-CPT-AM $(10 \mu \mathrm{M}, 30 \mathrm{~min})$. C Interaction of ACADL and $\mathbf{D}$ its phosphoresidues regulation by Epac1 and CaMKIl. Cardiomyocytes were preincubated or not with either CE3F4 ( $20 \mu \mathrm{M}, 30 \mathrm{~min})$ or KN-93 (5 $\mu \mathrm{M}, 30 \mathrm{~min})$ and were stimulated or not with 8-CPT-AM $(10 \mu \mathrm{M}, 30 \mathrm{~min})$. E ACADL-dependent serine phosphorylation after CaMKII $\delta$ silencing (siCaMKIII) or not (siCT) following 8-CPT-AM stimulation ( $10 \mu \mathrm{M}, 30 \mathrm{~min})$. F Regulation of ACADL phosphorylation by palmitate $(500 \mu \mathrm{M}, 30$ min). G Schematic representation of ACADL wild type (ACADL ${ }^{\mathrm{WT}}$ ) and mutant form (ACADL ${ }^{\mathrm{S} 193 \mathrm{~A}}$ ). $\mathbf{H}$, I Phosphorylation of ACADL in cells transfected with the indicated plasmids and stimulated with the indicated compounds. IgG and GAPDH were used as control for immunoprecipitation (IP). Input is a control of cell lysates. The immunoblots are representative of three independent experiments. J Determination of FAO by measuring radiolabeled $\mathrm{CO}_{2}$ produced by palmitate ${ }^{14} \mathrm{C}$ in cardiomyocytes expressing wild-type Epac1 (Epac1 ${ }^{\mathrm{WT}}$ ) and transfected with either ACADL ${ }^{\text {WT }}$ or ACADL ${ }^{\text {S193A }}$ $(n=14-17)$ The results are expressed as mean \pm SEM and were analyzed with a one-way ANOVA / Bonferroni post test. ${ }^{*} p<0.05$.

resistant to Epac1-induced FAO reduction, as shown by the increased amounts of radiolabelled $\mathrm{CO}_{2}$ compared to cells expressing ACADL ${ }^{\mathrm{WT}}$ (Fig. 5J). Altogether, these data indicate that Epac1-CaMKII axis targets enzymes of the FAO to decrease FA breakdown through the $\beta$-oxidation spiral.

\section{Epac1 regulates ATP synthase activity}

The reducing equivalents (NADH and FADH) resulting from the FAO deliver electrons through the distinct complexes of the electron transport chain (ETC) to build up a proton gradient used in fine by the ATP synthase to generate ATP from ADP [1]. 
A

\begin{tabular}{|c|c|}
\hline Spot ID & 507 \\
\hline Ratio (Vol) & 3,6 \\
\hline Regulation & Up \\
\hline ProteinID & $\begin{array}{c}\text { ATP synthase subunit } \\
\text { alpha, mitochondrial }\end{array}$ \\
\hline Score & 542 \\
\hline
\end{tabular}

C

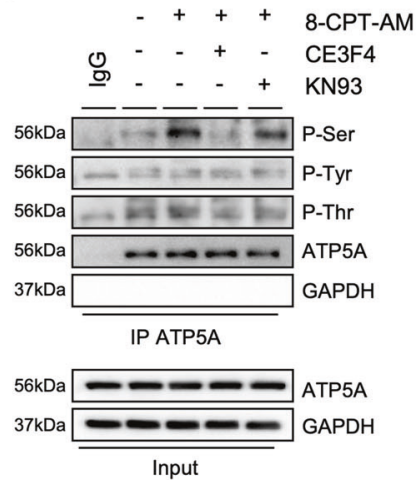

$\mathbf{F}$
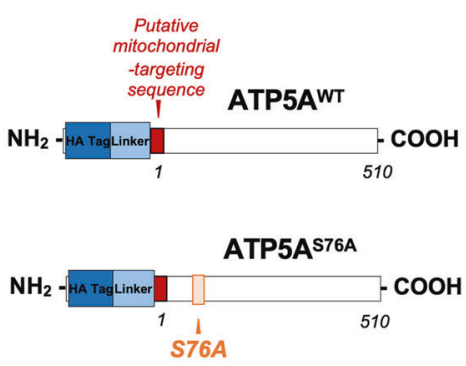

H

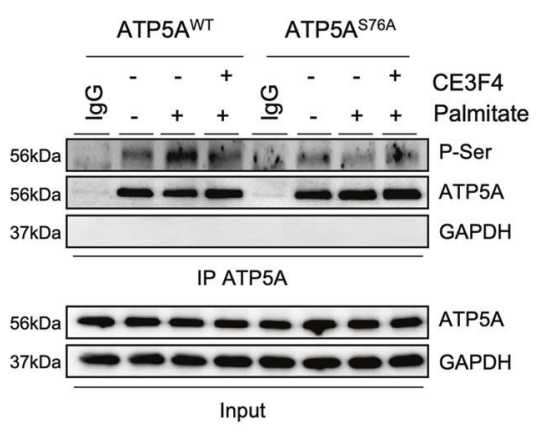

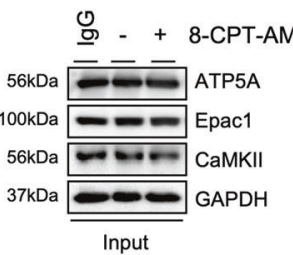

D

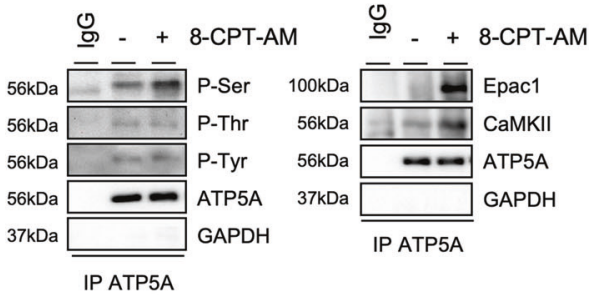

E

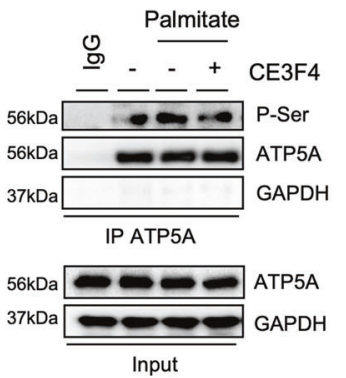

G

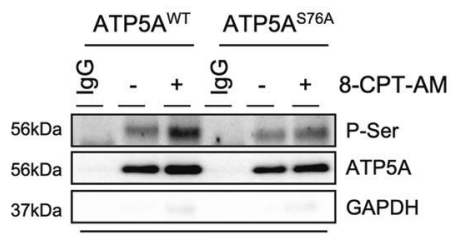

IP ATP5A

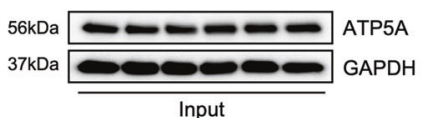

I

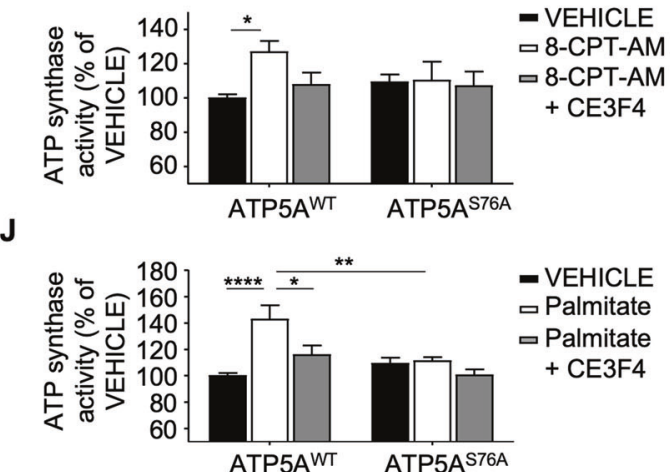

Fig. 6 Epac1 phosphorylates the a subunit of ATP synthase through CaMKII and regulates its activity. A Phosphoproteome results. The ratio of volume value is indicated in the table. The change in the intensity of the protein spot (507) is indicated as increased (up) in the stimulated 8-CPT-AM (10 $\mathrm{MM}, 10 \mathrm{~min})$ vs. control. ID, identity. B Representative immunoblots showing the interaction of ATP5A with Epac1 or CaMKII, or ATP5A phosphorylation level in cardiomyocytes stimulated or not with 8-CPT-AM (10 $\mu$ M, 30 min). C, D ATP5A phosphorylation in cells preincubated or not with either CE3F4 $(20 \mu \mathrm{M}, 30 \mathrm{~min})$ or $\mathrm{KN}-93(5 \mu \mathrm{M}, 30 \mathrm{~min})$ and stimulated or not with either $8-\mathrm{CPT}-\mathrm{AM}(10 \mu \mathrm{M}$, $30 \mathrm{~min})$ or palmitate $(500 \mu \mathrm{M}, 30 \mathrm{~min})$. E ATP5A-dependent serine phosphorylation after CaMKII $\delta$ silencing (siCaMKIIS) or not (siCT) following 8-CPT-AM stimulation $(10 \mu \mathrm{M}, 30 \mathrm{~min})$. F Schematic representation of ATP5A wild type (ATP5A ${ }^{\text {WT }}$ ) and mutant form (ATP5A ${ }^{\text {S76A }}$ ). G, $\mathbf{H}$ Phosphorylation of ATP5A ${ }^{\mathrm{WT}}$ or ATP5A $\mathrm{S}^{\mathrm{S} 6 \mathrm{~A}}$ in cells pretreated or with CE3F4 $(20 \mu \mathrm{M}, 30$ min) and stimulated or not with 8-CPT-AM $(10 \mu \mathrm{M}, 30 \mathrm{~min})$ or palmitate $(500 \mu \mathrm{M}, 30 \mathrm{~min})$. The immunoblots are representative of three independent experiments. IgG and GAPDH were used as control for IP. Input is a control of cell lysates. I, J ATP synthase activity in cardiomyocytes transfected with the indicated plasmids and pretreated as in $(\mathbf{G}, \mathbf{H})(n=6)$. The results are expressed as mean \pm SEM and were analyzed with a two-way ANOVA/Bonferroni post test. ${ }^{*} p<0.05,{ }^{* *} p<0.01,{ }^{* * * *} p<0.0001$ vs control group or indicated value.

Interestingly, the a subunit of ATP synthase (ATP5A) was revealed in Epac1 phosphoproteome (Fig. 6A). Epac1 activation increased ATP5A, Epac1, and CaMKII complex formation as well as ATP5A Ser-specific phosphorylation (Fig. 6B).
Pharmacological inhibition of Epac1 or CaMKII with CE3F4 or KN-93 blocked 8-CPT-AM-increased ATP5A Ser phosphorylation level (Fig. 6C). Similarly, palmitate-induced ATP5A phosphorylation was prevented by CE3F4 indicating that palmitate 


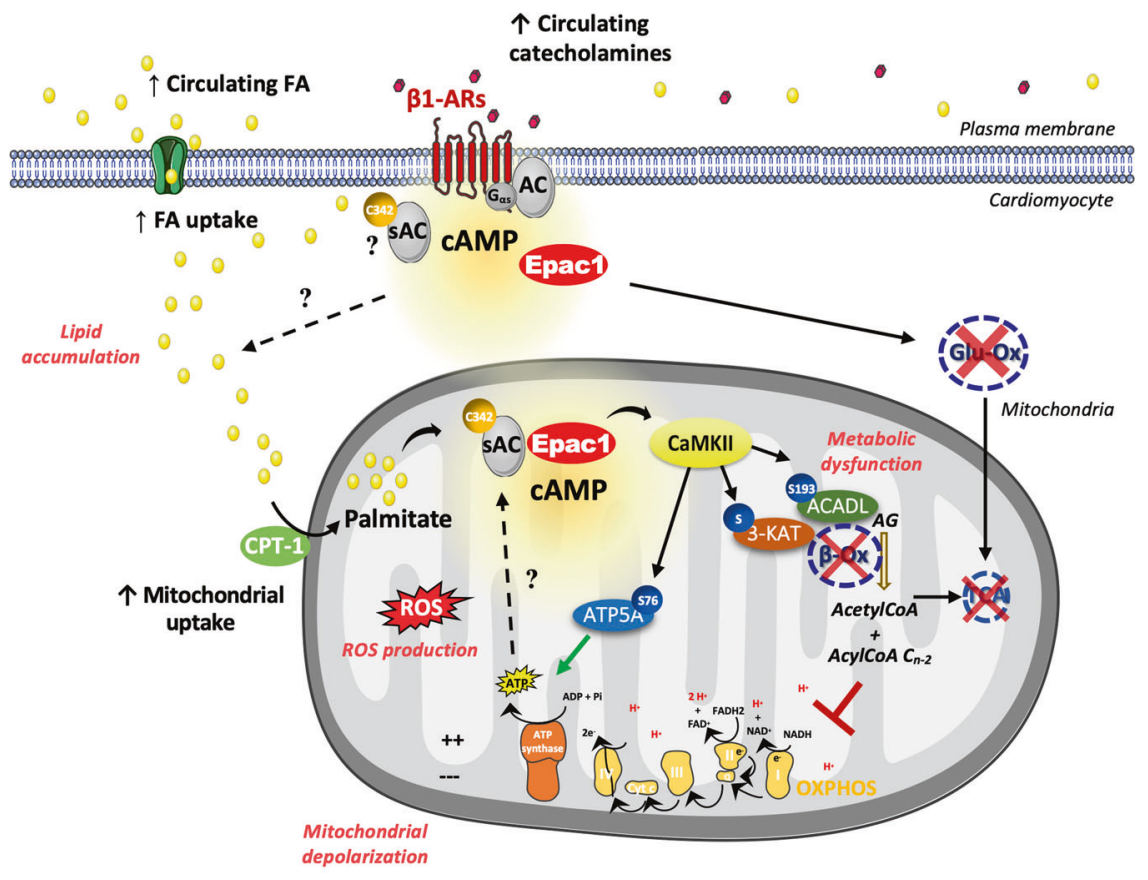

\section{Altered metabolic flexibility \\ Mitochondrial dysfunction}

Fig. 7 Schema illustrating a working hypothesis of Epac1 signaling during metabolic stress. In the setting of a metabolic stress, circulating levels of FA and catecholamines are increased and promote FA uptake inside the cardiomyocyte. Palmitate positively regulates Epac1 activity by soluble adenylyl cyclase (sAC) palmitoylation. Epac1 dysregulates FA metabolism (mitochondrial uptake and $\beta$-ox) via the regulation of CPT$1, \beta$-ox enzymes, and ATP synthase by CaMKIII-dependent phosphorylation. Thus, Epac1 disrupts the balance between the mitochondria uptake of FA and its consumption leading to accumulation of lipid intermediates, metabolic dysfunctions (ROS production, cell death, lipid accumulation, decreased oxidative metabolism), lipotoxicity, thereby affecting metabolic flexibility and cardiac functions. Epac1 exchange protein directly activated by cAMP, FA fatty acid, cAMP cyclic AMP, CaMKII Ca ${ }^{2+} /$ calmodulin-dependent protein kinase II, $\beta 1$-ARs $\beta 1$-adrenergic cardiac receptors, $\beta$-ox $\beta$-oxidation, Glu-ox glucose oxidation, CPT-1 cartinine palmitoyltransferase, ATP5A $\alpha$ subunit of ATP synthase, ACADL long-chain fatty acid acyl-CoA dehydrogenase, 3-KAT 3-ketoacyl-CoA thiolase, TCA tricarboxylic acid cycle, OXPHOS mitochondrial oxidative phosphorylation.

regulates the phosphorylation state of ATP5A via Epac1 (Fig. 6D).

A bioinformatic analysis of the ATP5A sequence identified a potential phosphorylation site on Ser76 for CaMKIII isoform. As expected, transfection of SiCaMKII $\delta$ or a mutated form of ATP5 on Ser76 (ATP5A ${ }^{\text {S76A }}$ ) prevented Epac1-mediated ATP5A serine phosphorylation (Fig. $6 \mathrm{E}, \mathrm{F}, \mathrm{G}$ ). The ATP5A $\mathrm{S}^{\mathrm{57A}}$ mutant was also effective in blocking the effect of palmitate on the phosphorylation level of ATP5AWT like CE3F4 (Fig. 6H). This effect also translated on ATP synthase activity (Fig. 6l, J). Finally, we assessed ATP synthase activity in WT and Epac1 ${ }^{-/-}$hearts subjected to chronic HFD. In-gel activity analysis showed that Epac1 genetic inhibition preserved a higher complex $\mathrm{V}$ activity and oligomerization after 15 weeks of HFD compared to WT mice (Supplementary Fig. 8) suggesting that Epac1 inhibition allows to maintain ATP production during chronic metabolic stress. Altogether, these data showed that Epac1-CaMKII axis is involved in the regulation of ATP synthase activity leading to an impaired mitochondrial energetics.

\section{DISCUSSION}

In this work, we provide novel insights into the role of CAMPEpac1 in cardiac myocytes (Fig. 7). Specifically, we demonstrate that a saturated FA palmitate regulates Epac1 activity by stimulating CAMP production via SAC palmitoylation at a highly conserved Cys 342 residue. Once activated by lipid overload, Epac1 enhanced CPT-1 activity and blocked lipid oxidation through
CaMKII-dependent phosphorylation of FAO enzymes. On the contrary, Epac1 inhibition increased FAO and prevented palmitate-induced mitochondrial dysfunction. Furthermore, Epac1-CaMKII axis interacted with the ATP5A and regulated ATP synthase activity, most likely to impair mitochondrial energetics during lipid overload.

Here, we characterized an unsuspected molecular mechanism that regulated the intracellular production of CAMP. Indeed, we found that SAC was activated by palmitoylation leading to increased cAMP production and Epac1 activation. Palmitoylation is a reversible lipid modification regulating many proteins [30]. For instance, palmitoylation of the voltage-gated sodium channel, Nav1.5 or the ryanodine receptor have been shown to affect their activity and ion homeostasis in cardiomyocytes [31-33]. Here we provide evidence that $S A C$ palmitoylation represents a novel signal transduction mechanism that is modulated by dietdependent signals and may impact the regulation of cell fate. Aberrant SAC palmitoylation turns on an Epac1-dependent activation of CaMKIl, which promotes mitochondrial dysfunction and cardiomyocyte death during chronic lipid overload. Importantly, we identified C342 as a conserved SAC palmitoylation substrate regulating $S A C$ activity. Loss of palmitoylation at this crucial residue prevented intracellular cAMP elevation and Epac1 activation in response to palmitate. At this time, it is still unclear how palmitoylation of C342 impacts SAC activity. The complete conservation of this cysteine among $S A C$ orthologs suggests that palmitoylation of this residue may serve as an important 
10

regulatory role in SAC activity. Of note, C342 is located in the C2 catalytic region of SAC and at the proximity of the ATP substrate binding site and $\mathrm{HCO}^{-}$regulatory binding site [26]. Therefore, this specific post-translational modification at C342 could impact SAC configuration, thus enhancing the interaction of the substrate with $\mathrm{SAC}$ and/or stimulating the effect of $\mathrm{HCO}_{3}{ }^{-}$on $\mathrm{SAC}$ activity.

Our study also identified novel mechanisms that contribute to cardiomyocyte lipotoxicity. First, we characterized a novel role of CAMP in the regulation of FA mitochondrial uptake. Indeed, we found that Epac1 enhanced CPT-1 activity, a key enzyme regulating mitochondrial FA uptake, which are then degraded in the $\beta$-oxidation pathway for the production of ATP [34]. It is known that prolonged lipid overload enhanced mitochondrial ROS generation, which is followed by reduced mitochondrial respiration and ATP synthesis (decreased mitochondrial FAO) [28]. We believe that the stimulating effect of Epac1 on CPT-1 activity promotes mitochondrial FA overload and contributes to lipotoxicity due to the inability of mitochondria to entirely catabolize increased amounts of FA. Accordingly, a previous study showed that cardiac-selective inhibition of CPT-1B, the predominant CPT-1 isoform expressed in the myocardium attenuated pathological cardiac remodeling and improved cardiac function in obese mice [35]. Yet, a selective $\beta$-blocker, metoprolol decreased CPT-1 activity and ameliorated cardiac contractility in dogs with heart failure further supporting the beneficial effect of CPT-1 inhibition in myocardial function [36]. In addition, cytosolic lipid accumulation may be due to a higher FA uptake by cardiomyocytes compared to FA mitochondrial uptake. Therefore, one cannot exclude that Epac1 may also regulate other key proteins involved in the transport of FA into the cardiomyocyte such as the plasma membrane-associated protein, FA translocase (also known as CD36), and FA transport protein 1 (FATP1).

Besides its effect on ROS production and mitochondrial potential, Epac1 may also influence cardiomyocyte death by altering the balance of mitochondrial dynamics (fusion/fission). Indeed, Yang and colleagues recently showed that Epac1-induced cardiomyoblast apoptosis in the context of hypoxia correlated with a change in the expression of key proteins involved in mitochondrial dynamics such as dynamin related protein 1 (DRP1) and mitofusin 2 (MFN2) [37]. However, in the context of lipid overload, mitochondrial morphology modification may not be a primary event in cell death. Indeed, Tsushima and collaborators reported that scavenging mitochondrial ROS prevented palmitateinduced dysregulation of mitochondrial dynamics in cardiomyocytes [28]. Therefore, the lipotoxic effect of palmitate is mainly mediated through Epac1-promoted ROS production and mitochondrial dysfunction.

Second, we characterized Epac1 as a novel regulator of oxidative metabolism in mitochondria. Pharmacological inhibition of Epac1 increased FAO in palmitate-treated cardiomyocytes under lipid overload condition. Mitochondrial Epac1 interacted with two $\beta$-oxidation enzymes, ACADL and 3-KAT to modify their phosphorylation state via CaMKII thereby decreasing FA breakdown through the $\beta$-oxidation pathway. Therefore, Epac1 activation decreases FAO degradation and together with its stimulating effect on FA uptake it facilitates intracellular accumulation of lipid intermediates thus causing mitochondrial dysfunction, ROS production and cardiomyocyte death. These findings suggest that enhancing mitochondrial FAO may prevent obesity induced cardiomyopathy. Consistently, a recent study showed that increasing mitochondrial FAO by genetic deletion of acetyl-CoA carboxylase 2 that enhanced FAO by relieving the inhibition of CPT-1 was sufficient to prevent the development of obesity induced cardiomyopathy, in part via the inhibition of mitochondrial dysfunction [38]. On the contrary, inhibiting the increase of myocardial FAO exacerbated cardiac damage in obese mice [39]. Taken together, these data suggest that Epac1 inhibition may represent a therapeutic opportunity for lipotoxic cardiomyopathy associated with obesity and diabetes.
Interestingly, we found that Epac1 pharmacological blockade not only increased ECAR, a key component of glycolysis rate but also prevented $\mathrm{FAO}$-induced glucose oxidation inhibition in lipid overload condition. Hence, Epac1 inhibition could reduce glucotoxicity arising from accelerated sugar-related modifications, such as O-GlyNAcylation and advanced glycation end products (AGE) formation. Altogether, these results show that Epac1 inhibition allows a better metabolic flexibility of cardiomyocytes in the context of lipid overload.

In our study, we reported that during a metabolic stress Epac1 interacted with the a subunit of ATP synthase, ATP5A and activated ATP synthase activity via a CaMKII $\delta$-dependent phosphorylation of ATP5A at Ser76. Accordingly, a previous study showed that inhibition of O-GlcNAcylation on ATP5A decreased ATP synthase activity and subsequent ATP production [40] indicating that post-translational modifications of ATP5A may strongly impact the activity of this enzyme. The functional consequence of Epac1-induced ATP synthase activity has yet to be investigated but one could hypothesize that this effect of Epac1 could stimulate the production of ATP, which in turn could stimulate SAC activity to enhance Epac1 activation and its detrimental role in cardiomyocytes. Alternatively, increased activation of ATP synthase by Epac1 could accelerate mitochondrial depolarization by disrupting the proton gradient that was not restored because of deficits in reduced equivalents. In line with this hypothesis, we demonstrated here that Epac1 induced CaMKII-dependent phosphorylation of FAO enzymes such as ACADL to decrease FAO and consequently the substrates that fueled mitochondrial oxidative phosphorylation.

Interestingly, we identified a novel regulatory signaling of FAO and complex $\mathrm{V}$ of the respiratory chain since we provided evidence that mitochondrial CaMKII, mainly the CaMKII $\delta$ isoform acted as a key downstream effector of Epac1 in the regulation of mitochondrial ACADL, 3-KAT, and ATP synthase. Of particular importance, we demonstrated that the regulatory effect of CaMKII on ACADL and ATP5A occurred via serine-dependent phosphorylation. This novel biological action of CaMKII in the mitochondrial compartment is in line with a previous study showing that (1) CaMKII had a mitochondrial localization, and (2) mitochondrial Epac1-CaMKII axis regulated the activity of the isocitrate dehydrogenase 2, a critical mitochondrial enzyme of the tricarboxylic acid (TCA) cycle [16]. Consistent with our data, previous work indicated that CaMKII contributed to the lipotoxicity of palmitate in cardiac-derived $\mathrm{H} 9 \mathrm{C} 2$ cells [41]. At present, further work is required to understand how Epac1 activates CaMKII in mitochondria and whether Rap-GTPase, a direct effector of Epac1, is involved in Epac1-CaMKII signalosome. Downstream effectors of CaMKII may also converge on key metabolic regulators AMP-activated protein kinase, and peroxisome proliferator-activated receptor gamma coactivator-1a, which interconnect with CAMP-Epac1 pathway [42].

In summary, we here identify a novel signaling pathway by which CAMP-Epac1 axis controls mitochondrial $\beta$-oxidation and metabolic flexibility in cardiomyocyte. We propose a model whereby, Epac1 disrupts the balance between mitochondrial FA uptake and oxidation leading to lipid accumulation, mitochondrial dysfunction, and cardiomyocyte death during lipid overload conditions. Epac1 inhibition allows a better management of substrates and energy within cardiomyocyte and therefore could have potential therapeutic implications for obesity induced cardiomyopathy.

\section{MATERIALS AND METHODS}

\section{Animals}

Mice were housed in a pathogen-free facility and all animal experiments were approved by the Animal Care and Use Committees of the University of Toulouse. Epac1-deficient mice $\left(\mathrm{EpaC1}^{-1-}\right)$ have been engineered in our laboratory as previously described [43]. 


\section{Cardiac lipotoxicity mice model}

Mice were placed either on a high-fat diet (HFD, 60\% saturated fat, 20\% proteins, and $20 \%$ carbohydrate, ResearchDiet, Broogarden, Denmark) or a normocaloric diet (ND, 10\% lipids, 20\% proteins, and 70\% carbohydrate SSNIFF Spezialdiäten GmBH, Germany) ad libidum for 15 weeks. The animals were housed on a day/night cycle $12 \mathrm{~h} / 12 \mathrm{~h}$ with unlimited access to food and water.

\section{Human heart tissues}

Human samples were collected in the Cardiac Surgery Department of the Toulouse University Hospital, France, and stored in liquid nitrogen. Samples used in this study were right atrial appendage tissue, retrieved after cardio-pulmonary bypass weaning, at the site of the venous cannula withdrawal scar. Obese status was defined as a body mass index over $30 \mathrm{Kg} / \mathrm{m}^{2}$, non-obese status was defined as a body mass index under $25 \mathrm{Kg} / \mathrm{m}^{2}$. The two groups were homogeneous concerning the main cardiac pathology, the preoperative left ventricle ejection fraction, and the presence or the absence of atrial fibrillation.

\section{Cell culture}

All procedures were performed in accordance with the Guide for the care and use of laboratory animals and the veterinary committee has been informed of the cardiac myocyte isolation protocol used. All media, sera, and antibiotics used in cell culture were purchased from Invitrogen.

Neonatal rat ventricular myocytes (NRVM) isolation. Neonatal SpragueDawley rats of 1-2 days old were euthanized by decapitation. The heart was excised and the atria were removed. Primary culture of NRVMs was subsequently performed as previously described [44]. The ventricles were pooled, and the ventricular cells were dispersed by digestion with collagenase II (Worthington) and pancreatin (Life Technologies, Inc.). The cell suspension was purified by centrifugation through a discontinuous Percoll gradient to obtain myocardial cell cultures with $>95 \%$ myocytes. The cardiomyocytes were plated at a density of $5-6 \times 10^{4}$ cells $/ \mathrm{cm}^{2}$ in Dulbecco's modified Eagle's medium (DMEM, Gibco) 4,5 g/L Glucose + glutamax/medium 199 (4:1) supplemented with $10 \%$ horse serum, $5 \%$ fetal bovine serum, glutamine, and antibiotics (plating medium), and allowed to attach overnight. The cells were then washed twice with DMEM/medium 199 and further incubated in DMEM/medium 199 supplemented with glutamine and antibiotics (maintenance medium) in the presence or absence of various agents.

Adult murine ventricular cardiomyocytes (AMVC) isolation. After intraperitoneal injection of pentobarbital $(300 \mathrm{mg} / \mathrm{kg})$ and heparin $(150 \mathrm{U})$, the hearts from WT and $\mathrm{Epac1}^{-1-}$ were rapidly isolated and placed in ice-cold Tyrode calcium free buffer $(130 \mathrm{mM} \mathrm{NaCl}, 5.4 \mathrm{mM} \mathrm{KCl}, 1.4 \mathrm{mM} \mathrm{MgCl}, 0.4 \mathrm{mM}$ $\mathrm{NaH}_{2} \mathrm{PO}_{4}, 4.2 \mathrm{mM}$ HEPES, $10 \mathrm{mM}$ glucose, $20 \mathrm{mM}$ taurine, and $10 \mathrm{mM}$ creatine monohydrate, $\mathrm{pH}$ 7.2). The heart was quickly excised, and the aorta was cannulated for retrograde perfusion in a Langendorff apparatus at a constant flow rate of $3 \mathrm{~mL} / \mathrm{min}$ at $37^{\circ} \mathrm{C}$. Primary culture of AMVCs was subsequently performed as previously described [16]. The heart was perfused for 6-8 min with Tyrode buffer, followed by Tyrode buffer containing $12.5 \mu \mathrm{M} \mathrm{CaCl} 2$ and $0.5 \mathrm{mg} / \mathrm{mL}$ Liberase $^{\mathrm{TM}}$ medium Thermolysin (Roche Diagnostics, Meylan, France) for $9 \mathrm{~min}$. Once the enzymatic digestion of the heart completed, the left ventricle was dissected, minced with scissors and homogenized with a pipette in Tyrode buffer containing $12.5 \mu \mathrm{M} \mathrm{CaCl}$ and $5 \%$ bovine serum albumin (Sigma-Aldrich, France) to stop the enzymatic reaction. Dispersed myocytes were then filtered through a $100 \mu \mathrm{m}$ mesh and allowed to sediment by gravity for $10 \mathrm{~min}$. The supernatant was removed and centrifuged for $1 \mathrm{~min}$ at $10 \times g$. The pellet was resuspended in Tyrode buffer containing $12.5 \mu \mathrm{M} \mathrm{CaCl} 2$. The calcium concentration was increased gradually from $12.5 \mu \mathrm{M}$ to $1 \mathrm{mM}$ in five steps over $\sim 20 \mathrm{~min}$. Freshly isolated cardiomyocytes were plated for $2 \mathrm{~h}$ on $35 \mathrm{~mm}$ laminin $(10 \mu \mathrm{g} / \mathrm{mL})$-coated tissue culture dishes in M199 complete medium (M199 medium with added $100 \mathrm{IU} / \mathrm{mL}$ penicillin, $2 \mathrm{mM}$ L-carnitine, $5 \mathrm{mM}$ creatine, and $5 \mathrm{mM}$ taurine). After this period of attachment, the medium was changed and cells were incubated overnight at $37^{\circ} \mathrm{C}$ in a humidified atmosphere of $5 \% \mathrm{CO}_{2}$ and air. The culture protocol yielded an average of $80 \%$ rodshaped myocytes at a plating density of 50 cells $/ \mathrm{mm}^{2}$ that were viable at $\mathrm{pH} 7.2$ for $48 \mathrm{~h}$. Experiments were performed the day following isolation and culture.

\section{Plasmid constructs and transfection}

The mutant form of Epac1 deleted for its putative mitochondrial-targeting sequence (Epac1 ${ }^{\Delta 2-37}$ ) was producted by mutagenesis in a previous study
[16]. The plasmid encoding the wild-type mitochondrial soluble adenylate cyclase $\left(s A C^{W T}\right)$ was provided by Dr. Gergö Szanda. The expression vector (pCMV3) encoding wild-type forms of ATP5A and ACADL enzymes were purchased from SinoBiological Inc. The mutant form of $S A C\left(S A C^{C 342 A}\right)$, $\mathrm{ACADL}^{\mathrm{S} 193 \mathrm{~A}}$, and ATP5A ${ }^{\mathrm{S} 76 \mathrm{~A}}$ were producted by mutagenesis (QuickChange Site-Directed Mutagenesis Kit, Agilent Technologies). Primary neonatal rat cardiomyocytes were transfected using Lipofectamine 2000 (Invitrogen) according to the manufacturer's instructions.

\section{Reagents and antibodies}

The highly membrane-permeant Epac1 agonist 8-(4-Chlorophenylthio)-2'O-methyladenosine-3', 5'-cyclic monophosphate, acetoxymethyl ester (8-CPT-AM) and its respective control (AM) were purchased from BioLog (Bremen, Germany). The Epac1 inhibitor CE3F4 was synthesized according to the methods published previously [23]. The following commercially available inhibitors were used: SAC inhibitor, KH7 (Cayman Chemical, Bertin Pharma, Montigny-le-Bretonneux, France); CaMKII inhibitor, KN-93 (Sigma-Aldrich, France); Cartinine palmitoyltranferase CPT-1 inhibitor, etomoxir (Sigma-Aldrich, France); ATP synthase inhibitor, oligomycin (Sigma-Aldrich, France); mitochondrial respiratory chain complex I inhibitor, rotenone (Sigma-Aldrich, France); and mitochondrial respiratory chain complex III inhibitor, antimycin A (Sigma-Aldrich, France). Carbonyl cyanide 4- (trifluoromethoxy)phenylhydrazone (FCCP), a protonophore decoupling the respiratory chain, D-Glucose and 2-deoxyglucose, BSA solution, sodium palmiate and BSA-Oleic acid (oleate) were purchased from Sigma-Aldrich. BSA-palmitic acid (palmitate) was produced and supplied by Dr. Cedric Moro (I2MC, France). Primary antibodies for immunoblot were obtained from the following sources: Epac1 (\#4155), Rap1 (\#4938), Myc-Tag (\#2276) (all at 1/1000) and GAPDH (\#2118, at 1/5000) from Cell Signaling (Ozyme, Montigny-le-Bretonneux, France). ACADL, ATP5A, and 3-KAT were immunoprecipitated using an antibody against Myc-Tag (\#2276, Cell Signaling). Primary antibodies against phosphoserine (\#ab9332), phosphotyrosine (\#ab10321), phosphothreonine (\#ab9337), CaMKII (\#ab181052), ADCY10 (\#ab82854), ACADL (\#ab128566), ATP5A (\#ab14748), and 3-KAT (\#ab230667) were from Abcam (all at $1 / 1000)$ and tubulin (\#T8203, at 1/5000) from Sigma-Aldrich. Unrelevant antibody for immunoprecipitation control (IgG1 Isotype Control, \#5415) was from CellSignaling. OXPHOS antibody cocktail was purchased from Mitoscience. All media, sera, and antibiotics used in cell culture were purchased from Invitrogen.

\section{Seahorse Agilent XFe24 experiments}

Mitochondrial respiration. Oxygen consumption rate (OCR) was measured with a Seahorse XFe24 analyzer (Agilent). ARVMs were seeded in 24-well Seahorse assay plates in complete medium and, after $2 \mathrm{~h}$ of cell attachment, the medium was replaced with XF basal medium supplemented with $10 \mathrm{mM}$ glucose, $4 \mathrm{mM}$ glutamine, and $1 \mathrm{mM}$ sodium pyruvate (pH 7.4). Plates were incubated for an additional $1 \mathrm{~h}$ at $37^{\circ} \mathrm{C}$ in a $\mathrm{CO}_{2}$-free incubator. The hydrated wells of the sensor cartridge were then loaded with $1 \mu \mathrm{M}$ oligomycin (port A), $1 \mu \mathrm{M}$ carbonyl cyanide 4- (trifluoromethoxy) phenylhydrazone (FCCP) (port B) and $1 \mu \mathrm{M}$ antimycin $\mathrm{A}+1 \mu \mathrm{M}$ rotenone (port C). The data were analyzed using the Seahorse Wave software.

FA oxidation. Oxidation of FA in mitochondria was measured with the Seahorse Agilent technology. After cell adhesion, complete medium was replaced with XF basal medium supplemented with $4 \mathrm{mM}$ glutamine and $0.5 \mathrm{mM}$ carnitine (for CPT-1 function) ( $\mathrm{pH}$ 7.4). Plates were incubated for an additional $1 \mathrm{~h}$ at $37^{\circ} \mathrm{C}$ in a $\mathrm{CO}_{2}$-free incubator. The hydrated wells of the sensor cartridge were then loaded with $2 \mu \mathrm{M}$ oligomycin (port $\mathrm{A}$ ), $2 \mu \mathrm{M}$ FCCP (port B) and $4 \mu \mathrm{M}$ antimycin $\mathrm{A}+2 \mu \mathrm{M}$ rotenone (port C). Fifteen minutes before the start of the measurement, the cells were treated with $40 \mu \mathrm{M}$ of etomoxir. At the time of starting the measurement, $500 \mu \mathrm{M}$ palmitate is added. The data were analyzed using the Seahorse Wave software.

Glycolysis function. Extracellular acidification rate (ECAR) was determined with a Seahorse XFe24 analyzer (Agilent) that allows measuring mitochondrial glucose oxidation. After cell adhesion, plating medium was replaced with a XF basal medium supplemented with $4 \mathrm{mM}$ L-glutamine $\left(\mathrm{pH}\right.$ 7.4). Plates were incubated for an additional $1 \mathrm{~h}$ at $37^{\circ} \mathrm{C}$ in a $\mathrm{CO}_{2}$-free incubator. The hydrated wells of the sensor cartridge were then loaded with $25 \mathrm{mM}$ D-glucose (port A), $2 \mu \mathrm{M}$ oligomycin (port B), and $50 \mathrm{mM}$ 2-deoxyglucose (port C). Data were analyzed using the Seahorse Wave software. 


\section{Mitochondrial ROS measurement}

Cells were incubated with $5 \mu \mathrm{M}$ of MitoSOX ${ }^{\mathrm{TM}}$ Red probe (Thermo Scientific) for $10 \mathrm{~min}$ to $37^{\circ} \mathrm{C}$, protected from light and then washed with PBS. Red fluorescence was measured at $580 \mathrm{~nm}$ after excitation of the probe at $510 \mathrm{~nm}$ using a fluorimeter.

\section{SiRNA experiments}

Rat CaMKII $\delta$-specific siRNA and control siRNA (Horizon Discovery) were resuspended at $20 \mu \mathrm{M}$ in distilled water. One day after plating, primary cardiomyocytes were transfected were transfected with DharmaFECT ${ }^{\circledR}$ Duo (Thermo Scientific) using $100 \mathrm{nM}$ of siRNA per well.

\section{Assessment of mitochondrial membrane potential $(\Delta \Psi \mathrm{m})$}

$\Delta \psi_{\mathrm{m}}$ was monitored using the fluorescence dye $5^{\prime}, 6,6^{\prime}$-tetrachloro1,1',3,3'-tetraethyl-benzamidazolocarbocyanin iodide (JC-1, Enzo Life Sciences, Villeurbanne, France). Cells were incubated with $5 \mu \mathrm{M} \mathrm{JC}-1$ in M199 complete medium at $37^{\circ} \mathrm{C}$ for $15 \mathrm{~min}$ and then washed with PBS. Excitation of the dye was performed at $496 \mathrm{~nm}$ and emission signal ratio $(590 / 530)$ was normalized to that measured under VEHICLE condition.

\section{Rap activation assay}

Rap1 pull-down experiments were performed using the Rap1 binding domain of Ral-GDS fused to GST as described previously [23]. Cells were starved for $1 \mathrm{~h}$ before stimulation in serum-free MEM containing penicillinstreptomycin (1\%). After transfection, cells were lysed in radioimmune precipitation assay buffer $(50 \mathrm{mM}$ Tris- $\mathrm{HCl} \mathrm{pH}=7.5,500 \mathrm{mM} \mathrm{NaCl}, 20 \mathrm{mM}$ $\mathrm{MgCl}_{2}, 0.5 \%$ deoxycholic acid, $0.1 \%$ SDS, $1 \%$ Triton X-100, $1 \mathrm{mM}$ PMSF, protease, and phosphatase inhibitors), and $500 \mu \mathrm{g}$ of protein was incubated with Ral-GDS coupled to glutathione-Sepharose beads (Amersham Biosciences) for $1 \mathrm{~h}$ at $4{ }^{\circ} \mathrm{C}$. The beads were then washed three times in washing buffer $(50 \mathrm{mM}$ Tris- $\mathrm{HCl} \mathrm{pH}=7.5,150 \mathrm{mM} \mathrm{NaCl}, 20 \mathrm{mM} \mathrm{MgCl}$, $1 \%$ Triton X-100, $0.1 \mathrm{mM}$ PMSF, protease, and phosphatase inhibitors) and then resuspended and heated for $10 \mathrm{~min}$ at $95^{\circ} \mathrm{C}$ in Laemmli to perform immunoblots.

\section{Acyl-biotin exchange method (ABE)}

ABE experiments experiments were performed as previously described [25]. Briefly, cell lysates were incubated overnight at $4{ }^{\circ} \mathrm{C}$ with $\mathrm{N}$-ethylmaleimide (Thermo Scientific) to block free cysteine and to ensure complete cysteine alkylation. The next day, the insoluble component was removed after centrifugation at $13,500 \times g$ for $5 \mathrm{~min}$. The proteins were precipitated with chloroform/methanol to remove excess chemicals. After the last centrifugation, proteins were dissolved in $1 \mathrm{~mL}$ of PBS containing $1 \%$ SDS. The soluble protein was divided into two equal groups. The first group was resuspended in a solution containing $0.7 \mathrm{M}$ hydroxylamine $\mathrm{NH}_{2} \mathrm{OH}, 1 \mathrm{mM}$ biotin, $0.2 \%$ Triton X-100 supplemented with protease inhibitors. The second one was treated with $200 \mathrm{mM}$ Tris, $1 \mathrm{mM}$ biotin, $0.2 \%$ Triton X-100 supplemented with antiproteases. Hydroxylamine (HAM, Sigma-Aldrich) is a powerful reducing agent that cleaves the palmitate from cysteines to allow biotinylation. Sites blocked by N-ethylmaleimide (Thermo Scientific) were not cleaved. The lysates were also treated simultaneously with the biotin-HPDP reagent (Thermo Scientific). The remaining chemicals were removed using chloroform/methanol precipitation and resolubilized in PBS containing 1\% Triton X-100 and 0.2\% SDS. The biotinylated proteins were captured by streptavidin-Sepharose beads (GE-Healthcare) for $2 \mathrm{~h}$ at room temperature. Then, beads were washed four times with PBS containing 1\% Triton X-100; $0.2 \%$ SDS and then resuspended and heated for $10 \mathrm{~min}$ at $95^{\circ} \mathrm{C}$ in Laemmli to perform immunoblots.

\section{Immunoprecipitation}

Cardiomyocytes were mixed overnight at $4{ }^{\circ} \mathrm{C}$ with $5 \mu \mathrm{g}$ of appropriated antibody in buffer containing $20 \mathrm{mM}$ Tris, pH 7.5, $150 \mathrm{mM} \mathrm{NaCl}, 1 \mathrm{mM}$ EDTA, 1\% Triton X-100, protease, and phosphatase cocktail inhibitors (Roche). Purification steps were performed with protein A/G agarose according to the manufacturer's instructions (SantaCruz).

\section{Western blot}

Once denatured, the samples were deposited and separated by SDS-PAGE electrophoresis ( $20 \mathrm{~min}$ at $80 \mathrm{~V}$ then $2 \mathrm{~h}$ at $130 \mathrm{~V}$ at room temperature). The proteins were then transferred to a PVDF membrane, previously activated in $100 \%$ ethanol for $5 \mathrm{~min}$, using the Trans-blot transfer apparatus (20 V,
$1 \mathrm{~A})$ for $30 \mathrm{~min}$. The membrane was then blocked in a solution of TBSTween 20-BSA 3\% minimum $1 \mathrm{~h}$ at room temperature. After incubation with the primary antibody overnight at $4{ }^{\circ} \mathrm{C}$, a secondary antibody or Protein G-HRP (dilution 1:5000, Biorad) was added for $90 \mathrm{~min}$ at room temperature. Only the membranes having fixed samples resulting from immunoprecipitation experiments were incubated with Protein G-HRP in order to overcome the labeling of heavy and light chains of the denatured antibody. After washing with TBS-Tween, membranes were revealed by chemiluminescence using GE-Healthcare Amersham ECL Kit and readed with BioradChemDocTM XRS+. Quantification was performed using the ImageLab software and normalized to a household protein (GAPDH (Cell Signaling) or Tubulin (Sigma-Aldrich)).

\section{Capillary western blot}

Epac1 protein quantification was achieved by capillary western blot analysis using the ProteinSimple Jess system with 12-230 kDa Jess separation module capillary cartridges (ProteinSimple, SantaClara, CA, USA). A mouse monoclonal antibody specific for Epac1 (Cell Signaling) was used (1:50). Anti-mouse detection module for Jess (ProteinSimple) kit included Luminol-S, peroxide, antibody diluents, streptavidin coupled horseradish peroxidise, anti-mouse secondary antibody, and a protein normalization reagent. Sample proteins ( $\mathrm{ng}$ per each condition) were allowed to separate via the capillary technology and were analysed based on chemiluminescence, which was transformed into digital images depicting bands as observed in western blots. The abundance of Epac1 and total proteins were determined using Compass software (ProteinSimple). The normalized data are expressed as Epac1 signal intensity over total proteins signal intensity.

\section{Lactate dehydrogenase (LDH) release}

$\mathrm{LDH}$ release in the cell culture medium was measured according to the manufacturer's instructions (LDH-Cytotoxicity Assay Kit II, Abcam).

\section{MTT assay}

Cells were plated in $100 \mu \mathrm{L}$ in 96-well flat bottom plates and then exposed to tested agents. At the end of treatment, $10 \mu \mathrm{L}$ of $5 \mathrm{mg} / \mathrm{mL}$ MTT (3-[4,5dimethylthiazol-2-yl]-2,5-diphenyltetrazolium bromide; thiazolyl blue, Sigma-Aldrich) solution in PBS were added to each well for $3 \mathrm{~h}$. After removal of the medium, $50 \mu \mathrm{L}$ of DMSO were added to each well to dissolve the formazan crystals. The absorbance at $550 \mathrm{~nm}$ was determined using a plate reader (TECAN infinite F500). Triplicate wells were assayed for each condition.

\section{cAMP quantification}

Analysis of the total cellular CAMP content was performed using the CAMP direct Enzyme Immunoassay Kit (Enzo Life Sciences). Briefly, supernatants were removed and cells were treated with $0.1 \mathrm{M} \mathrm{HCl}$ for $10 \mathrm{~min}$ at RT and were centrifuged $\geq 600 \times g$ to pellet the cellular debris. The supernatant was either assayed immediately or stored frozen $\left(-20^{\circ} \mathrm{C}\right)$ for later analysis according to the manufacturer's instructions.

\section{Palmitate oxidation}

Cardiomyocytes were incubated for $3 \mathrm{~h}$ with $\left[1-{ }^{14} \mathrm{C}\right]$ palmitate $(1 \mu \mathrm{Ci} / \mathrm{ml}$; Perkinelmer, Boston, MA) and nonlabeled (cold) palmitate at a final concentration of $100 \mu \mathrm{M}$. Palmitate was coupled to a FA-free BSA in a molar ratio of 5:1. Following incubation, ${ }^{14} \mathrm{CO}_{2}$ and ${ }^{14} \mathrm{C}$-ASM (acid soluble metabolites) were measured as previously described [45]. Briefly, assayed medium was transferred into a custom-made Teflon 48-well trapping plate. The plate was clamped and sealed, and perchloric acid was injected through the perforations in the lid into the medium, which drives $\mathrm{CO}_{2}$ through the tunnel into an adjacent well, where it is trapped in $1 \mathrm{~N} \mathrm{NaOH}$. Following trapping, the media was spun twice and ${ }^{14} \mathrm{C}$-ASM measured by scintillation counting. Aliquots of $\mathrm{NaOH}$ and medium were transferred into scintillation vials, and radioactivity was measured on a multipurpose scintillation counter (LS 6500; Beckman Coulter). All assays were performed in triplicates, and data normalized to cell protein content.

\section{Carnitine palmitoyltranferase 1 (CPT-1) activity}

Cells were lysed in a buffer containing $20 \mathrm{mM}$ Tris $\mathrm{pH} 7.5,150 \mathrm{mM} \mathrm{NaCl}$, $1 \mathrm{mM}$ EDTA, Triton 1\%, protease inhibitors, and phosphatases. CPT-1 activity was determined by spectrophotometry by measuring the amount of the reduced form of coenzyme $\mathrm{A}(\mathrm{COA}-\mathrm{SH})$ released using the Ellman 
reagent (5,5'-dithio-bis (2-nitrobenzoic acid (DTNB), Sigma-Aldrich) as previously described [46]. In the presence of DTNB, CoA-SH colors the medium in yellow. Briefly, cells were incubated with a reaction buffer (20 mM HEPES pH 7.4, $220 \mathrm{mM}$ sucrose, $40 \mathrm{mM} \mathrm{KCl}, 1 \mathrm{mM}$ EGTA, $0.1 \mathrm{mM}$ DTNB) and the absorbance was measured for 90 min (every $30 \mathrm{~s}$ ) at $412 \mathrm{~nm}$ after adding $40 \mu \mathrm{M}$ palmitoyl-CoA and $5 \mathrm{mM} \mathrm{L-carnitine.} \mathrm{CPT-1} \mathrm{activity} \mathrm{was}$ defined in $\mathrm{nmol} \mathrm{CoA}-\mathrm{SH}$ released/min/mg protein and expressed as a percentage of the control condition.

\section{Assessment of ATP synthase activity}

ATP synthase activity was determined with the ATP Synthase Enzyme Activity Microplate Assay Kit (Abcam) in accordance with the manufacturer's instructions. Activities were measured in $\mathrm{pM} / \mathrm{mg}$ of protein and expressed as a percentage of the control condition.

\section{Blue native PAGE (BN-PAGE) analysis of mitochondrial supercomplexes (SCs) and in-gel activity}

BN-PAGE was performed using the Native PAGE TM system (Invitrogen). Briefly, $100 \mu \mathrm{g}$ of isolated cardiac mitochondria were solubilized by digitonin ( $8 \mathrm{~g} / \mathrm{g}$ protein) for $20 \mathrm{~min}$ on ice, then centrifuged at $20,000 \times g$ at $4{ }^{\circ} \mathrm{C}$ for $10 \mathrm{~min}$. After centrifugation, coomassie blue G-250 (Invitrogen) was added to the supernatant to obtain a dye/detergent concentration ratio of $1 / 4$ and then the protein was loaded into a $4-12 \%$ nondenaturing polyacrylamide gel (Invitrogen). After electrophoresis, the complexes and SCs can be visualized by a simple Coomassie staining and destaining method. For in-gel activity analysis, $35 \mathrm{mM}$ Tris, $270 \mathrm{mM}$ glycine, $14 \mathrm{mM}$ $\mathrm{MgSO}_{4}, 10 \mathrm{mM}$ ATP, and $0.2 \% \mathrm{~Pb}\left(\mathrm{NO}_{3}\right)_{2}$ in water were added to the $4-12 \%$ nondenaturing polyacrylamide gel after electrophoresis and incubated for overnight at RT. The reaction was stopped with $50 \%$ of methanol. Appearance of transparent/silver bands is indicative of complex $V$ activity. Since the bands are transparent, the gel should be inverted (to black background) after scanning to visualize the activity clearly. The activity was determined by analyzing the inverted black bands using Image J.

\section{Lipid accumulation}

Red oil is a Sudan-type fat-soluble dye that attaches specifically to neutral lipids (triglycerides). After fixation with $4 \%$ paraformaldehyde for $10 \mathrm{~min}$, cardiomyocytes were incubated with Oil Red O working solution $160 \%$ of $0.5 \%$ red oil ( $0.94 \mathrm{~g}$ dissolved in $150 \mathrm{~mL}$ isopropyl alcohol) diluted in $40 \%$ of distilled water), for $20 \mathrm{~min}$ at RT. After washing with PBS, slides were mounted with Vectashield in an aqueous medium and then cells were observed under a phase contrast fluorescence microscope.

\section{Quantification and statistical analysis}

Data are presented as mean or fold increase \pm SEM. No statistical analysis was used to predetermine sample size. Estimates were made based on our previous experience, experimental approach, availability of the samples. Prespecified exclusion criteria in cell experiments is absence of response in the palmitate only condition for expected effects (cell death, ROS production, mitochondrial membrane potential). No exclusion criteria were applied in the others experiments. All experiments were performed with at least three independent biological replicates (exact number is reported in figures' legend). Cages housing mice were randomly assigned to experimental or control diet. Investigators were blinded to the group of the individual animals or patients during the outcome assessments. Analyses were performed using Prism 7 (GraphPad Software). Data distribution within groups/conditions were validated by the Shapiro-Wilk normality test. Multiple comparisons were performed with one-way or twoway analysis of variance (ANOVA) as appropriate followed by post-hoc test with Bonferroni correction for multiple comparison, if normality test was not passed the nonparametric Kruskal-Wallis test was performed. Parametric unpaired two-tailed $t$-test was used for comparisons of two groups, if normality test was not passed the nonparametric Mann-Whitney test was used. Two-tailed $p$-value were used. Asterisk $\left(^{*}\right)$ indicates $p<0.05$; ${ }^{* *} p<0.01 ; * * * p<0.001 ;{ }^{* * * *} p<0.0001 ;$ ns, not significant. Differences in means were considered statistically significant at $p<0.05$.

\section{REFERENCES}

1. Lopaschuk GD, Ussher JR, Folmes CD, Jaswal JS, Stanley WC. Myocardial fatty acid metabolism in health and disease. Physiol Rev. 2010;90:207-58.
2. Goldberg IJ, Trent CM, Schulze PC. Lipid metabolism and toxicity in the heart. Cell Metab. 2012;15:805-12.

3. Jia G, Hill MA, Sowers JR. Diabetic cardiomyopathy: an update of mechanisms contributing to this clinical entity. Circ Res. 2018;122:624-38.

4. Fukushima A, Lopaschuk GD. Cardiac fatty acid oxidation in heart failure associated with obesity and diabetes. Biochim Biophys Acta. 2016;1861:1525-34.

5. Beavo JA, Brunton LL. Cyclic nucleotide research-still expanding after half a century. Nat Rev Mol Cell Biol. 2002;3:710-8.

6. Halls ML, Cooper DMF. Adenylyl cyclase signalling complexes-pharmacological challenges and opportunities. Pharmacol Ther. 2017;172:171-80.

7. Pozdniakova S, Ladilov Y. Functional significance of the adcy10-dependent intracellular CAMP compartments. J Cardiovasc Dev Dis. 2018;5:29

8. Di Benedetto G, Gerbino A, Lefkimmiatis K. Shaping mitochondrial dynamics: the role of cAMP signalling. Biochem Biophys Res Commun. 2018;500:65-74.

9. Acin-Perez R, Salazar E, Kamenetsky M, Buck J, Levin LR, Manfredi G. Cyclic AMP produced inside mitochondria regulates oxidative phosphorylation. Cell Metab. 2009;9:265-76.

10. Acin-Perez R, Gatti DL, Bai Y, Manfredi G. Protein phosphorylation and prevention of cytochrome oxidase inhibition by ATP: coupled mechanisms of energy metabolism regulation. Cell Metab. 2011;13:712-9.

11. Di Benedetto G, Scalzotto E, Mongillo M, Pozzan T. Mitochondrial Ca(2)(+) uptake induces cyclic AMP generation in the matrix and modulates organelle ATP levels. Cell Metab. 2013;17:965-75.

12. Aneja A, Tang WH, Bansilal S, Garcia MJ, Farkouh ME. Diabetic cardiomyopathy: insights into pathogenesis, diagnostic challenges, and therapeutic options. Am J Med. 2008:121:748-57.

13. Bockus LB, Humphries KM. CAMP-dependent protein kinase (PKA) signaling is impaired in the diabetic heart. J Biol Chem. 2015;290:29250-8.

14. Zhang F, Zhang L, Qi Y, Xu H. Mitochondrial cAMP signaling. Cell Mol Life Sci. 2016;73:4577-90.

15. Wang Z, Liu D, Varin A, Nicolas V, Courilleau D, Mateo P, et al. A cardiac mitochondrial CAMP signaling pathway regulates calcium accumulation, permeability transition and cell death. Cell Death Dis. 2016;7:e2198.

16. Fazal L, Laudette M, Paula-Gomes S, Pons S, Conte C, Tortosa F, et al. Multifunctional mitochondrial Epac1 controls myocardial cell death. Circ Res. 2017;120:645-57.

17. Laudette M, Zuo H, Lezoualc'h F, Schmidt M. Epac function and CAMP scaffolds in the heart and lung. J Cardiovasc Dev Dis. 2018;5(1):9

18. Kawasaki H, Springett GM, Mochizuki N, Toki S, Nakaya M, Matsuda M, et al. A family of CAMP-binding proteins that directly activate Rap1. Science. 1998;282:2275-9.

19. de Rooij J, Zwartkruis FJ, Verheijen MH, Cool RH, Nijman SM, Wittinghofer A, et al. Epac is a Rap1 guanine-nucleotide-exchange factor directly activated by cyclic AMP. Nature. 1998;396:474-7.

20. Robichaux WG 3rd, Cheng X. Intracellular CAMP sensor EPAC: physiology, pathophysiology, and therapeutics development. Physiol Rev. 2018;98:919-1053.

21. Wang H, Robichaux WG, Wang Z, Mei FC, Cai M, Du G, et al. Inhibition of Epac1 suppresses mitochondrial fission and reduces neointima formation induced by vascular injury. Sci Rep. 2016;6:36552.

22. Sparagna GC, Hickson-Bick DL, Buja LM, McMillin JB. A metabolic role for mitochondria in palmitate-induced cardiac myocyte apoptosis. Am J Physiol Heart Circ Physiol. 2000;279:H2124-2132.

23. Courilleau D, Bisserier M, Jullian JC, Lucas A, Bouyssou P, Fischmeister R, et al. Identification of a tetrahydroquinoline analog as a pharmacological inhibitor of the cAMP-binding protein Epac. J Biol Chem. 2012;287:44192-202.

24. Vliem MJ, Ponsioen B, Schwede F, Pannekoek WJ, Riedl J, Kooistra MR, et al. 8-pCPT-2'-O-Me-cAMP-AM: an improved Epac-selective cAMP analogue. Chembiochem. 2008;9:2052-4.

25. Wan J, Roth AF, Bailey AO, Davis NG. Palmitoylated proteins: purification and identification. Nat Protoc. 2007;2:1573-84.

26. Steegborn C. Structure, mechanism, and regulation of soluble adenylyl cyclasessimilarities and differences to transmembrane adenylyl cyclases. Biochim Biophys Acta. 2014;1842:2535-47. 12 Pt B

27. Noordali H, Loudon BL, Frenneaux MP, Madhani M. Cardiac metabolism-a promising therapeutic target for heart failure. Pharmacol Ther. 2018;182:95-114.

28. Tsushima K, Bugger $\mathrm{H}$, Wende AR, Soto J, Jenson GA, Tor AR, et al. Mitochondrial reactive oxygen species in lipotoxic hearts induce post-translational modifications of AKAP121, DRP1, and OPA1 that promote mitochondrial fission. Circ Res. 2018;122:58-73.

29. Zlobine I, Gopal K, Ussher JR. Lipotoxicity in obesity and diabetes-related cardiac dysfunction. Biochim Biophys Acta. 2016;1861:1555-68.

30. Spinelli M, Fusco S, Grassi C. Nutrient-dependent changes of protein palmitoylation: impact on nuclear enzymes and regulation of gene expression. Int J Mol Sci. 2018;19(12):3820 
31. Fuller W, Reilly L, Hilgemann DW. S-palmitoylation and the regulation of NCX1. Channels (Austin). 2016;10:75-77.

32. Chaube R, Hess DT, Wang YJ, Plummer B, Sun QA, Laurita K, et al. Regulation of the skeletal muscle ryanodine receptor $/ \mathrm{Ca}^{2+-}$ release channel RyR1 by S-palmitoylation. J Biol Chem. 2014;289:8612-9.

33. Pei Z, Xiao Y, Meng J, Hudmon A, Cummins TR. Cardiac sodium channel palmitoylation regulates channel availability and myocyte excitability with implications for arrhythmia generation. Nat Commun. 2016;7:12035.

34. Kim TT, Dyck JR. The role of CD36 in the regulation of myocardial lipid metabolism. Biochim Biophys Acta. 2016;1861:1450-60.

35. Zhang $Y$, Fang $X$, Dai M, Cao Q, Tan T, He W, et al. Cardiac-specific downregulation of carnitine palmitoyltransferase- $1 \mathrm{~b}$ (CPT- $1 \mathrm{~b})$ prevents cardiac remodeling in obese mice. Obesity (Silver Spring). 2016;24:2533-43.

36. Panchal AR, Stanley WC, Kerner J, Sabbah HN. Beta-receptor blockade decreases carnitine palmitoyl transferase I activity in dogs with heart failure. J Card Fail. 1998;4:121-6.

37. Yang $H$, Xue W, Ding $C$, Wang $C$, Xu B, Chen $S$, et al. Vitexin mitigates myocardial ischemia/reperfusion injury in rats by regulating mitochondrial dysfunction via Epac1-Rap1 signaling. Oxid Med Cell Longev. 2021;2021:9921982.

38. Shao D, Kolwicz SC Jr, Wang P, Roe ND, Villet O, Nishi K, et al. Increasing fatty acid oxidation prevents high-fat diet-induced cardiomyopathy through regulating Parkin-mediated mitophagy. Circulation. 2020;142:983-97.

39. Yan J, Young ME, Cui L, Lopaschuk GD, Liao R, Tian R. Increased glucose uptake and oxidation in mouse hearts prevent high fatty acid oxidation but cause cardiac dysfunction in diet-induced obesity. Circulation. 2009;119:2818-28.

40. Cha MY, Cho HJ, Kim C, Jung YO, Kang MJ, Murray ME, et al. Mitochondrial ATP synthase activity is impaired by suppressed O-GlcNAcylation in Alzheimer's disease. Hum Mol Genet. 2015;24:6492-504.

41. Zhong P, Quan D, Peng J, Xiong X, Liu Y, Kong B, et al. Role of CaMKII in free fatty acid/hyperlipidemia-induced cardiac remodeling both in vitro and in vivo. J Mol Cell Cardiol. 2017;109:1-16.

42. Park SJ, Ahmad F, Philp A, Baar K, Williams T, Luo H, et al. Resveratrol ameliorates aging-related metabolic phenotypes by inhibiting CAMP phosphodiesterases. Cell. 2012;148:421-33.

43. Laurent AC, Bisserier $M$, Lucas $A$, Tortosa $F$, Roumieux $M$, De Régibus $A$, et al. Exchange protein directly activated by CAMP 1 promotes autophagy during cardiomyocyte hypertrophy. Cardiovasc Res. 2015;105:55-64.

44. Morel E, Marcantoni A, Gastineau M, Birkedal R, Rochais F, Garnier A, et al. cAMPbinding protein Epac induces cardiomyocyte hypertrophy. Circ Res. 2005;97:1296-304.

45. Engeli S, Birkenfeld AL, Badin PM, Bourlier V, Louche K, Viguerie N, et al. Natriuretic peptides enhance the oxidative capacity of human skeletal muscle. J Clin Invest. 2012;122:4675-9.

46. Creus A, Ferreira MR, Oliva ME, Lombardo YB. Mechanisms involved in the improvement of lipotoxicity and impaired lipid metabolism by dietary alphalinolenic acid rich salvia hispanica $L$ (Salba) seed in the heart of dyslipemic insulin-resistant rats. J Clin Med. 2016;5:18

\section{ACKNOWLEDGEMENTS}

We acknowledge members of the UMS006 facilities, Xavier Sudre, and his staff for animal housing and Carine Pestourie, Marie-Laure Boizeau at in vivo imaging service (ENI) (UMS006/INSERM/UPS, Anexplo/Genotoul Plateform). We are grateful to Gergő Szanda for providing the plasmid encoding the wild-type mitochondrial SAC.

\section{AUTHOR CONTRIBUTIONS}

$\mathrm{FL}$ supervised the whole project. $\mathrm{ML}$ and $\mathrm{FL}$ performed study concept and design; $\mathrm{ML}$ carried out most of the experiments; YSM, GC, DB, IB, SB, LK, KF, DE, FT, AL, CB, BM,
$\mathrm{OL}$, and JMP contributed to several experiments; ML, YSM, GC, DB, JB, JF, AL, JMP, CM, and $\mathrm{FL}$ helped with data analyses and discussions; $\mathrm{ML}$ and $\mathrm{FL}$ performed development of methodology and wrote the original draft. All authors read and approved the final paper.

\section{FUNDING}

This work was supported by grants from Institut National de la Santé et de la Recherche Médicale, Agence Nationale de la Recherche (ANR-17-CE14-0014-01, ANR19-CE14-0038-01), Fondation de France (00066331/00096289), Fédération Française de Cardiologie, and Fondation pour la Recherche Médicale (Programme "Equipes FRM 2021").

\section{COMPETING INTERESTS}

The authors declare no competing interests.

\section{ETHICS APPROVAL AND CONSENT TO PARTICIPATE}

This study is in compliance with the Declaration of Helsinki. The Toulouse University Hospital local ethic committee has approved the procedure. A non-objection to research form was collected for each patient included. All animal procedures were performed in accordance with Institutional Guidelines on Animal Experimentation and with a French Ministry of Agriculture license and approved by local regulatory committee CEEA-122. Moreover, this investigation conformed to the Guide for the Care and Use of Laboratory Animals published by the Directive 2010/63/EU of the European Parliament.

\section{ADDITIONAL INFORMATION}

Supplementary information The online version contains supplementary material available at https://doi.org/10.1038/s41419-021-04113-9.

Correspondence and requests for materials should be addressed to F.L.

Reprints and permission information is available at http://www.nature.com/ reprints

Publisher's note Springer Nature remains neutral with regard to jurisdictional claims in published maps and institutional affiliations.

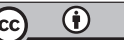

Open Access This article is licensed under a Creative Commons Attribution 4.0 International License, which permits use, sharing, adaptation, distribution and reproduction in any medium or format, as long as you give appropriate credit to the original author(s) and the source, provide a link to the Creative Commons license, and indicate if changes were made. The images or other third party material in this article are included in the article's Creative Commons license, unless indicated otherwise in a credit line to the material. If material is not included in the article's Creative Commons license and your intended use is not permitted by statutory regulation or exceeds the permitted use, you will need to obtain permission directly from the copyright holder. To view a copy of this license, visit http://creativecommons. org/licenses/by/4.0/.

(c) The Author(s) 2021 